\title{
The Succinoglycan Endoglycanase Encoded by exoK Is Required for Efficient Symbiosis of Sinorhizobium meliloti 1021 with the Host Plants Medicago truncatula and Medicago sativa (Alfalfa)
}

\author{
Hajeewaka C. Mendis, Clothilde Queiroux, Tess E. Brewer, Olivia M. Davis, Brian K. Washburn, and \\ Kathryn M. Jones \\ Department of Biological Science, Florida State University, Biology Unit I, 230A, 89 Chieftain Way, Tallahassee 32306-4370, \\ U.S.A.
}

Submitted 26 March 2013. Accepted 30 April 2013.

\begin{abstract}
The acidic polysaccharide succinoglycan produced by the nitrogen-fixing rhizobial symbiont Sinorhizobium meliloti 1021 is required for this bacterium to invade the host plant Medicago truncatula and to efficiently invade the host plant $M$. sativa (alfalfa). The $\beta$-glucanase enzyme encoded by exoK has previously been demonstrated to cleave succinoglycan and participate in producing the low molecular weight form of this polysaccharide. Here, we show that exoK is required for efficient $S$. meliloti invasion of both $M$. truncatula and alfalfa. Deletion mutants of exoK have a substantial reduction in symbiotic productivity on both of these plant hosts. Insertion mutants of exoK have an even less productive symbiosis than the deletion mutants with the host $M$. truncatula that is caused by a secondary effect of the insertion itself, and may be due to a polar effect on the expression of the downstream exoLAMON genes.
\end{abstract}

Sinorhizobium meliloti 1021 is a soil bacterium that establishes a nitrogen-fixing symbiosis with the host plants Medicago truncatula 'Jemalong A17', M. sativa (alfalfa), and other species of the Medicago and Melilotus genera (Gibson et al. 2008; Jones et al. 2007; Oldroyd et al. 2011; Udvardi and Poole 2013). These Medicago spp. are both agriculturally important forage crops and key model organisms for studying the symbiotic interaction between rhizobial bacteria and legume plant hosts. Under nitrogen-limiting conditions, S. meliloti induces the formation of nodules on host plant roots, invades and colonizes these nodules (Jones et al. 2007; Oldroyd et al. 2011), and then begins to convert or "fix" dinitrogen gas to ammonia, a form that the plant can use (Udvardi and Poole 2013). The invasion of host plant roots by rhizobial bacteria requires that multiple signals be exchanged between these symbiotic partners. Host plants produce flavonoid compounds that signal $S$. meliloti to produce a lipochitooligosaccharide called Nod factor (NF) (Peters et al. 1986). NF induces multiple responses in host plants, including curling of root hairs that

Corresponding author: K. M. Jones; Telephone: +1.850 .645 .8743 ; E-mail: kmjones@bio.fsu.edu

* The $\boldsymbol{e}$-Xtra logo stands for "electronic extra" and indicates that a supplementary table is published online.

(C) 2013 The American Phytopathological Society encloses bacterial cells within the curl and the induction of cell division in the root cortex which initiates the formation of the nodule primordium (Gage 2004; Oldroyd and Downie 2006). The bacteria invade and colonize the roots through tubules called infection threads that originate from microcolonies of bacteria trapped in the curls of the root hair cells (Jones et al. 2007; Timmers et al. 1999). These infection threads are progressive ingrowths of plant cell membrane containing a matrix composed of bacterial exopolysaccharides (EPS) and plant cell wall material (Gage 2004). Infection thread initiation and development require that $S$. meliloti propagate in the infection thread and produce both the EPS succinoglycan and NF (Klein et al. 1988). The infection threads in root hair cells are extended to the base of these cells, and new infection threads initiate at each cell layer, eventually delivering the bacteria to proliferating cells of the inner root cortex (Timmers et al. 1999; Voroshilova et al. 2009).

Succinoglycan is required for S. meliloti 1021 to induce infection thread development on its host plants, because it is the only symbiotically active EPS that this strain produces in sufficient quantities to mediate infection thread formation (Glazebrook and Walker 1989; Leigh and Lee 1988). S. meliloti 1021 strains that do not produce succinoglycan, such as the exoY mutant (Reed et al. 1991), are able to colonize plant surfaces and become enclosed within curled root hairs but they fail to initiate infection threads, and the few infection threads that are initiated are aborted (Cheng and Walker 1998). These aborted infections are associated with gene expression responses (Jones et al. 2008) and cytological responses (Niehaus et al. 1993) characteristic of plant defense reactions. It has not yet been determined how succinoglycan influences the conditions within the colonized curled root hair or why it is required for infection thread initiation and progression.

The structure of succinoglycan has been determined (Reinhold et al. 1994) and the biosynthesis of succinoglycan by S. meliloti 1021 has been studied extensively (Jones et al. 2007). The succinoglycan monomer is an octasaccharide composed of one galactose and seven glucose residues, with acetyl, succinyl, and pyruvyl modifications (Reinhold et al. 1994). The carboxylate groups of the succinyl and pyruvyl moieties render the polysaccharide acidic. The structure of the succinoglycan monomer along with the identity of the gene product that catalyzes each step in the biosynthetic pathway is shown in Figure 1A (Glucksmann et al. 1993a and b; Reinhold et al. 1994; Reuber and Walker 1993a and b). The undecaprenyl-phosphate galac- 
tose phosphotransferase encoded by exoY and the glycosyltransferases encoded by exoA, exoL, exoM, exoO, exoU, and exoW are required for production of complete succinoglycan (Glucksmann et al. 1993a and b; Long et al. 1988; Reed et al. 1991; Reuber and Walker 1993a and b). The exoH gene product transfers the succinyl substituents to the oligosaccharide (Leigh et al. 1987) while exo $V$ and exoZ add the pyruvyl and acetyl groups, respectively (Glucksmann et al. 1993b; Reuber and Walker 1993a and b). The glycanases encoded by exoK and exs $H$ cleave the succinoglycan polymer to produce the low molecular weight (LMW) fraction of succinoglycan (York and Walker 1998a). The gene organization of the exo genes on the $S$. meliloti pSymB plasmid is shown in Figure 1B (Finan et al. 2001).

Other rhizobial strains and species also produce acidic EPS that facilitate infection thread formation. Galactoglucan (EPSII), produced by S. meliloti SU47 and S. meliloti 8530 (S. meliloti

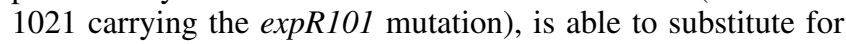
succinoglycan in facilitating infection thread formation on the host plant alfalfa but not on the host $M$. truncatula (Glazebrook and Walker 1989; Zhan et al. 1989). The acidic EPS produced by Rhizobium leguminosarum bv. viciae and $R$. leguminosarum bv. trifolii is required for infection thread formation by these symbionts on their plant hosts (Borthakur et al. 1986; Diebold and Noel 1989; Laus et al. 2005; van
Workum et al. 1995). It has also recently been demonstrated that Mesorhizobium loti mutants with defects in the later stages of EPS biosynthesis have greatly reduced efficiency in their symbiotic interaction with plant hosts of the genus Lotus (Kelly et al. 2013). In $S$. fredii NGR234, strains that do not produce symbiotic EPS are impaired in the interaction with the plant host Leucaena leucocephala (Staehelin et al. 2006). The symbiotic EPS produced by all of these rhizobial species are acidic, due to the presence of succinyl or pyruvyl modifications or the presence of glucuronic acid in the oligosaccharide chain (Aman et al. 1981; Djordjevic et al. 1986; Gil-Serrano et al. 1990; Jansson et al. 1977; Laus et al. 2005; O'Neill et al. 1991; Reinhold et al. 1994; Robertsen et al. 1981; Zhan et al. 1989).

The LMW fraction of $S$. meliloti succinoglycan has been proposed to be critical for this bacterium to initiate and maintain infection thread formation on its plant hosts (Battisti et al. 1992; Urzainqui and Walker 1992). In the studies that have reported rescue of host plant invasion by $S$. meliloti EPS-deficient mutants by coinoculation with purified EPS, this rescue has been associated with the LMW fraction of the EPS (Battisti et al. 1992; Urzainqui and Walker 1992). The LMW fraction is generated by cleavage of the succinoglycan chain to form exclusively monomers, dimers, and trimers of the octasaccharide repeating unit (Wang et al. 1999). Uncleaved, high molecular weight (HMW) succinoglycan is a very viscous material com-
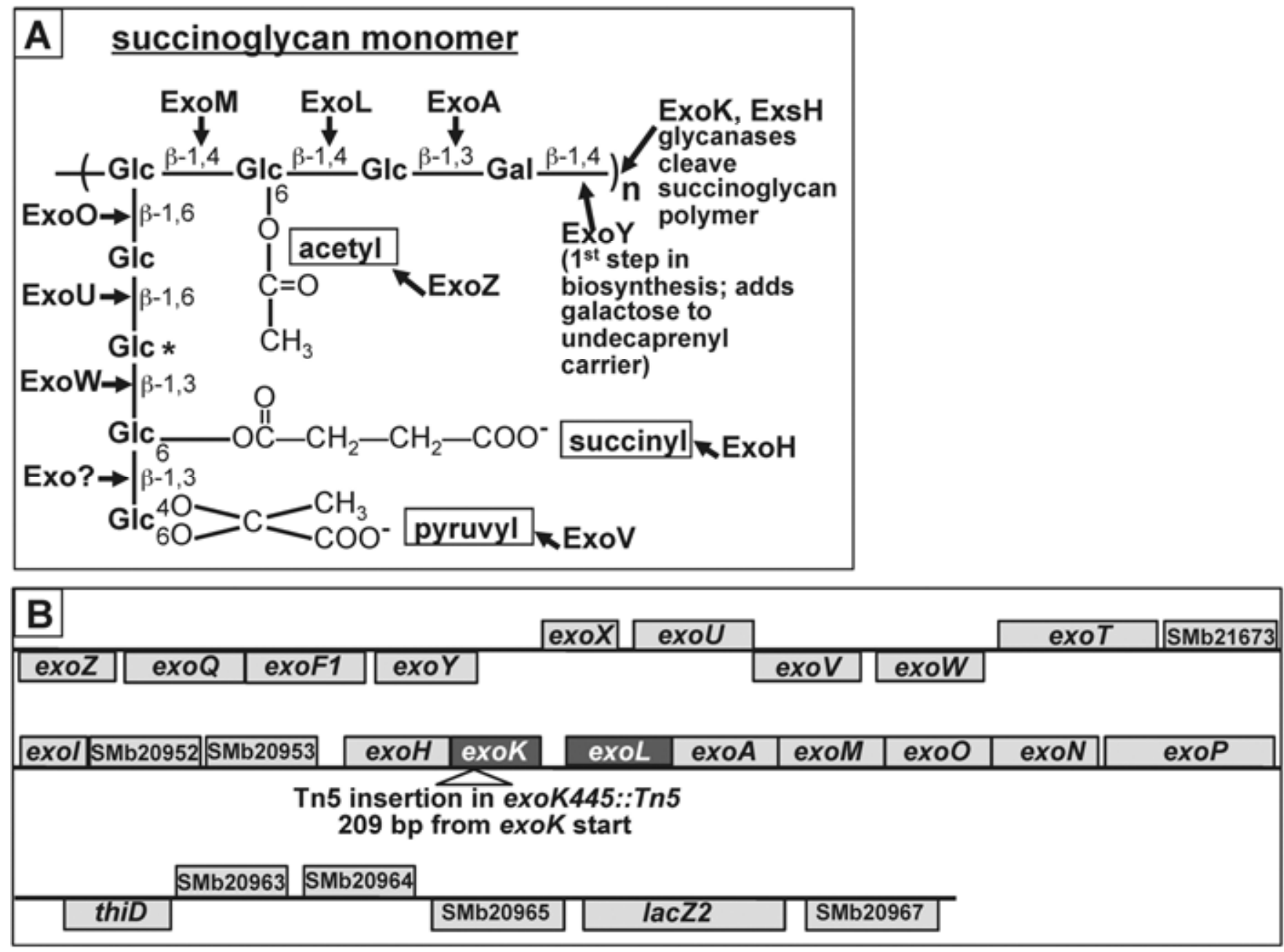

Fig. 1. A, Structure of the succinoglycan monomer, showing the sugar linkages and the positions of the acetyl, succinyl, and pyruvyl substituents (Glucksmann et al. 1993a and b; Reinhold et al. 1994; Reuber and Walker 1993a and b). A second succinylation site is marked with an asterisk (Wang et al. 1999). Glycanases encoded by the exoK and exs $H$ genes (glycosyl hydrolase family 16) cleave HMW succinoglycan into the LMW form (York and Walker 1997, 1998a). The glycosyltransferase encoded by the exoL gene adds the second glucose residue onto the growing oligosaccharide (Reuber and Walker 1993b). B, Organization of the succinoglycan biosynthesis genes on the S. meliloti 1021 pSymB plasmid (Galibert et al. 2001). 
posed of chains of hundreds of the octasaccharide repeating unit (Wang et al. 1999). Succinoglycan cleavage is performed by two $\beta$-glucanases of glycosyl hydrolase family 16 (GH16), encoded by the exoK and exsH genes (Glucksmann et al. 1993b; York and Walker 1997, 1998a). S. meliloti 1021 strains carrying transposon Tn5 or antibiotic-resistance cassette insertions in the exoK (Becker et al. 1993; Long et al. 1988; York and Walker 1997) and exsH (York and Walker 1997) genes produce a smaller amount of succinoglycan in the LMW form than does S. meliloti 1021 wild type (Sm1021[w.t.]). These S. meliloti exoK (Becker et al. 1993; Long et al. 1988; York and Walker 1997) and exsH (York and Walker 1997) insertion mutants have previously been reported to produce functional, nitrogenfixing nodules on the host plant alfalfa but the symbiotic phenotypes of these mutants were not quantified in these studies. In contrast, an $S$. fredii NGR234 strain carrying an $\Omega$-cassetteinsertion in the exoK gene, which encodes a $\beta$-glucanase $83 \%$ identical at the amino acid level to the one encoded by $S$. meliloti exoK (Altschul et al. 1997), forms no functional nodules and has an ineffective symbiosis with several of NGR234's plant hosts (Staehelin et al. 2006). Another set of observations may also provide evidence for the importance of LMW succinoglycan in host plant invasion. An exoH mutant, which produces succinoglycan without the succinyl modification, is completely blocked in host invasion (Cheng and Walker 1998) and cannot engage in a productive symbiosis (Leigh et al. 1987). This unsuccinylated succinoglycan produced by exoH transposoninsertion mutants cannot be cleaved by the ExoK and ExsH glycanases (York and Walker 1998b), and almost all of the polysaccharide produced by exoH mutants is in the HMW form. Because exoH mutants produce succinoglycan that is both unsuccinylated and exclusively in the HMW form, it is not clear at this point whether the symbiotic defects are due to the absence of the succinyl modification or to the absence of the LMW fraction of succinoglycan.

Because of the profound symbiotic defect of an $S$. fredii NGR234 exoK $\Omega$-cassette-insertion mutant, the demonstrated role of the exoK-encoded glycanase in generating LMW succinoglycan, and the evidence suggesting a critical role for the LMW fraction of succinoglycan in S. meliloti invasion of its hosts we have further examined the role of exoK in $S$. meliloti 1021 interaction with its host plants. We have determined the symbiotic phenotypes of the $S$. meliloti exoK445::Tn5 transposon insertion mutant and new deletion mutants of exoK on the model host plant Medicago truncatula A17 and the host plant alfalfa. Analysis of the deletion mutants demonstrates that loss of exoK in S. meliloti 1021 confers a significant loss of symbiotic efficiency.

\section{RESULTS}

\section{Symbiotic phenotypes of the exoK445::Tn5 mutant.}

The alfalfa symbiotic phenotype of the exoK445::Tn5 mutant was originally reported in Long and associates (1988) and confirmed as Fix + by York and Walker (1997) but the symbiotic phenotype was not characterized further. We have found that the exoK445::Tn5 mutant has a severe symbiotic defect on the plant host $M$. truncatula A17 and a moderate symbiotic defect on the plant host alfalfa. The symbiotic productivity measured by i) shoot length and ii) shoot fresh weight of both $M$. truncatula and alfalfa plants inoculated with the exoK445::Tn5 mutant compared with the Sm1021(w.t.) and with uninoculated plants is shown in Figure 2. The shoot weight of $M$. truncatula plants inoculated with the exoK445::Tn5 mutant is approximately 30\% that of plants inoculated with Sm1021(w.t.) (Fig. 2B). The alfalfa symbiotic productivity with exoK445::Tn5 is also lower than with Sm1021(w.t.) (Fig. 2A and B), although the effect is not as large as that seen with M. truncatula. The exoK445::Tn5 mutation also reduced the development of functional root nodules on $M$. truncatula, as shown by a decrease in the number of pink nodules (Fig. 2C). Pink color of the root nodules induced by rhizobial infection is due to the production of leghemoglobin by the host plant and is indicative of a functional symbiosis (Johnson and Vance 1981; Pladys and Vance 1993; Vance et al. 1980; Vasse et al. 1993). The larger number of white nodules on $M$. truncatula roots inoculated with the exoK445::Tn5 mutant indicates that nodule primordia are induced by this strain but the nodules are unable to develop and mature. In contrast, there is not a significant difference in the number of pink nodules formed on alfalfa roots by the exoK445::Tn5 mutant versus Sm1021(w.t.). This suggests that the symbiosis with $M$. truncatula is more sensitive to defects of the exoK445::Tn5 strain than is the symbiosis with alfalfa.

\section{The symbiotic defects of the exoK445::Tn5 mutant are not complemented by expression of exoK alone.}

Our next goals were to determine whether the $M$. truncatula symbiotic defect of the exoK445::Tn5 strain could be complemented by expression of exoK, and whether overexpression of exoK in Sm1021(w.t.) could lead to improved symbiotic productivity of this strain. There was a slight but significant increase in shoot growth and development of pink nodules by plants inoculated with Sm1021(w.t.) cells carrying the pExoK:: MSO4 overexpression construct relative to those carrying the negative control plasmid pMSO4 (Fig. 3A and B). However, we found that expression of exoK alone from the trp promoter in plasmid pMSO4 (Barnett et al. 2000) was not able to complement the $M$. truncatula shoot growth defect or the nodule development defect of the exoK445::Tn5 mutant (Fig. 3A and B). To determine whether the pExoK::MSO4 plasmid was expressing ExoK protein, we performed a Western blot with $\alpha$ ExoK antisera (provided by Graham C. Walker, Massachusetts Institute of Technology) (Fig. 3C). Both the Sm1021(w.t.) and exoK445::Tn5 strains carrying the pExoK::MSO4 plasmid produced a large quantity of ExoK protein when grown in MGS medium (York and Walker 1997) (Fig. 3C, lanes 3 and 5). The Sm1021(w.t.) strain carrying the negative control plasmid produced a moderate amount of ExoK protein (lane 2), while the exoK445::Tn5 mutant carrying the negative control plasmid produced no ExoK protein (lane 4). This indicates that ExoK protein is produced by the pExoK::MSO4 plasmid, at least in the free-living state, and that the lack of complementation of the exoK445::Tn5 symbiotic phenotypes is not due to a nonfunctional complementation construct. A second exoK expression construct was made in the stable, low-copy, pLAFR1based plasmid pRF771 (Chen et al. 2008; Egelhoff and Long 1985; Wells and Long 2002). The resulting plasmid, pExoK:: RF771, also failed to complement the exoK445::Tn5 defects in symbiotic productivity (Fig. 3D) and in nodule development (Fig. 3E). The pExoK::RF771 plasmid did not confer enhanced symbiotic phenotypes on the Sm1021(w.t.) strain.

\section{Expression of exoK complements the delayed Calcofluor-halo phenotype of the exoK445::Tn5 mutant strains.}

$S$. meliloti strains with mutations in exo $K$ are distinguished by delayed formation of a "halo" on agar medium containing the fluorescent dye Calcofluor (Becker et al. 1993; Long et al. 1988; York and Walker 1997). Calcofluor is used as an indicator of succinoglycan production because it fluoresces when bound to this polysaccharide (Wood 1980). LMW succinoglycan is able to diffuse away from growing $S$. meliloti cells into the surrounding Calcofluor-agar medium, producing a fluorescent halo around the cells, whereas HMW succinoglycan does not readily 
diffuse and remains closely associated with the growing cells (Leigh et al. 1987; Long et al. 1988). Because exoK mutants produce less LMW succinoglycan than does Sm1021(w.t.) (Becker et al. 1993; York and Walker 1997), there is a delay in the development of a Calcofluor halo around the exoK mutant cells (Long et al. 1988). We tested both exoK445::Tn5 strains and Sm1021(w.t.) strains expressing exoK from the pExoK:: RF771 plasmid to determine whether ExoK expression is suffi-
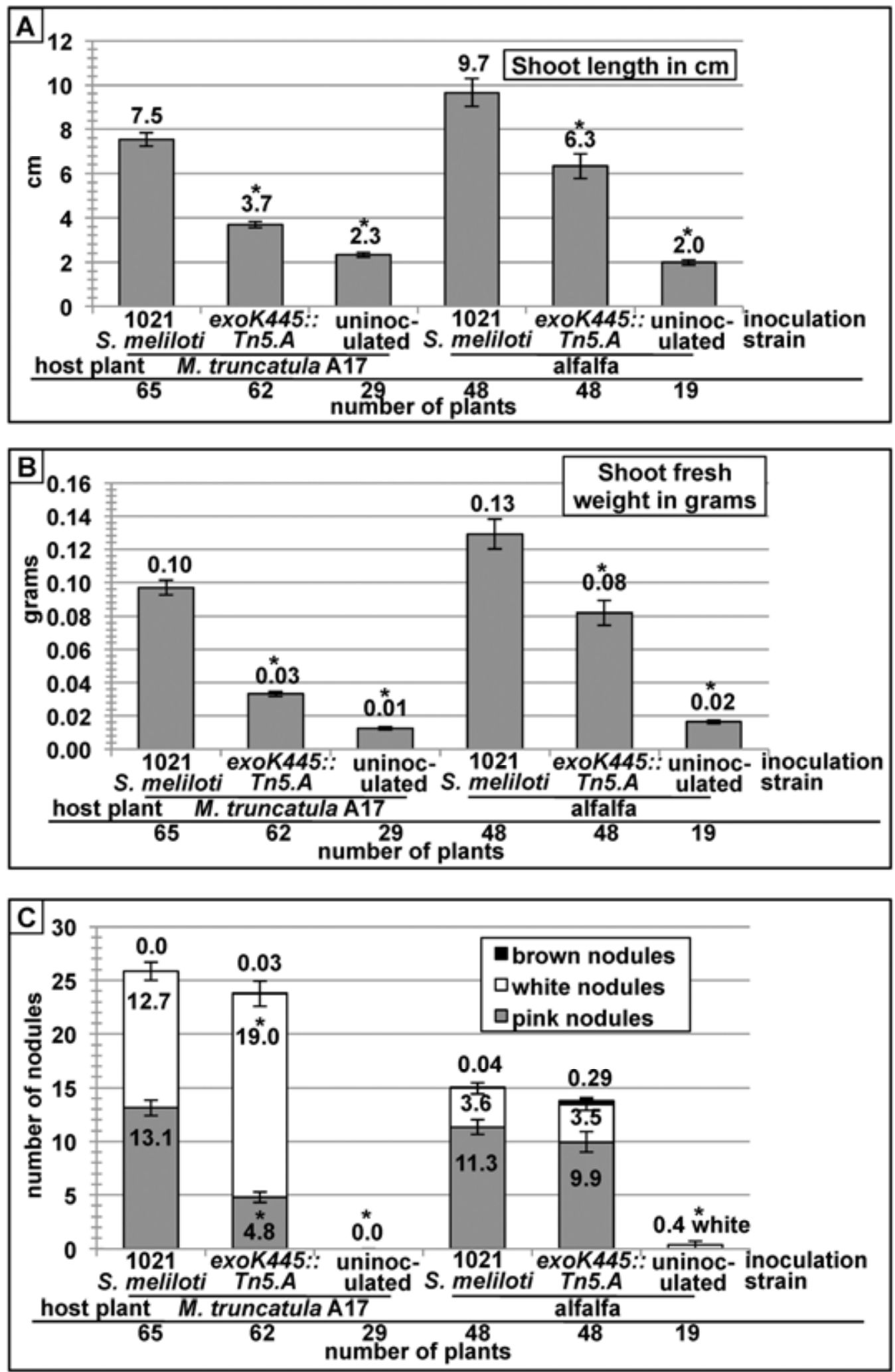

Fig. 2. The exoK445::Tn5 strain has reduced symbiotic productivity on Medicago truncatula and alfalfa, measured by A, shoot length or B, shoot fresh weight. C, Degree of nodule development, shown as the number of pink, functional nodules; white, nonfunctional nodules; and brown, necrotic nodules. $M$. truncatula A17 plants were grown for 7 weeks and alfalfa plants for 5 weeks. Asterisk $\left.*^{*}\right)$ denotes statistically significant difference from that plant species inoculated with the Sinorhizobium meliloti 1021 wild type reference strain. Data are averaged from two separate experiments for alfalfa and three separate experiments for M. truncatula. 

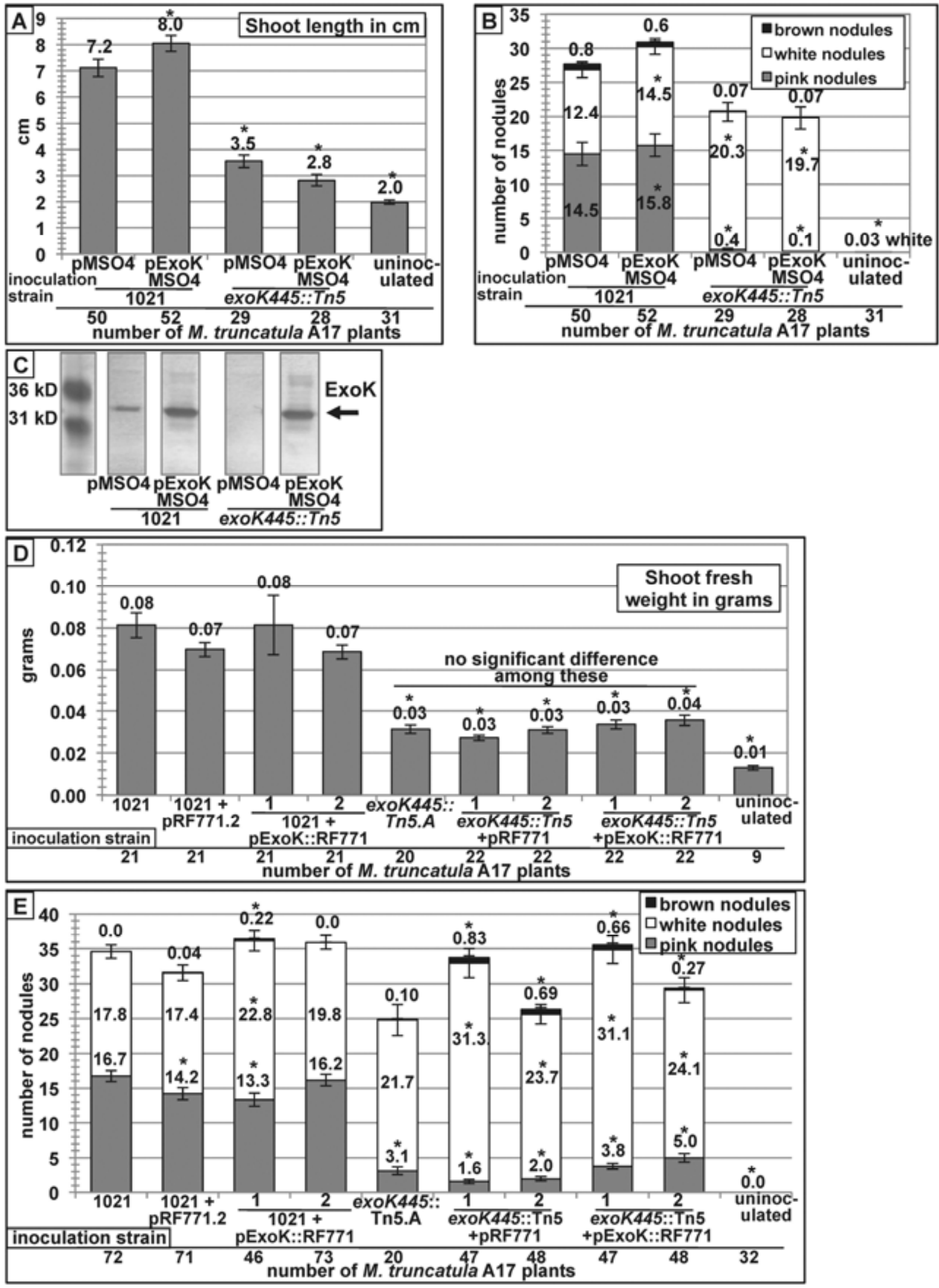

Fig. 3. ExoK expression alone does not complement the exoK445::Tn5 mutant symbiotic defect on Medicago truncatula A17. A, Plant height in centimeters and $\mathbf{B}$, number of pink, functional nodules demonstrate that exoK445::Tn5 is not complemented by expression of the exoK open reading frame. $\mathbf{C}$, Western blot probed with $\alpha$-ExoK antisera (provided by G. C. Walker, Massachusetts Institute of Technology) showing production of ExoK protein under the control of the trp promoter of the pMSO4 plasmid in Sinorhizobium meliloti strains. Expression of exoK alone in a second complementation construct (the pExoK::RF771 plasmid) also does not complement the D, reduced shoot weight or E, reduced number of pink, functional nodules of exoK445::Tn5 mutant strains. Values for independently isolated strains of each genotype are grouped together (e.g., 1021 + pExoK::RF771 "1" and "2"). Asterisk (*) denotes statistically significant difference from plants inoculated with $S$. meliloti 1021 wild type. Plant phenotype data are averaged from three separate experiments. 
cient to complement the exoK445::Tn5 delayed-halo phenotype in free-living cells. Development of the Calcofluor halo around Sm1021(w.t.) and exoK445::Tn5 cells imaged at 3-day intervals is shown in Figure 4A to D. As a comparison, the Calcofluordark phenotype of an exoY::Tn5 mutant is shown in Figure 4E. A magnified view of a representative Calcofluor halo outlined with a circle is shown in Figure 4F. The difference in Calcofluor-halo phenotypes between the strains is particularly evident after 9 days of growth (Fig. 4C). Sm1021(w.t.) cells with no plasmid and those carrying the pRF771 negative control plasmid have a prominent Calcofluor halo (Fig. 4C), whereas exoK445::Tn5 cells and exoK445::Tn5 + pRF771 cells have not yet developed a halo. Complementation of the delayed-halo phenotype of exoK445::Tn5 by the pExoK::RF771 plasmid is evident in the right-hand side of the same panel. Both Sm1021(w.t.)

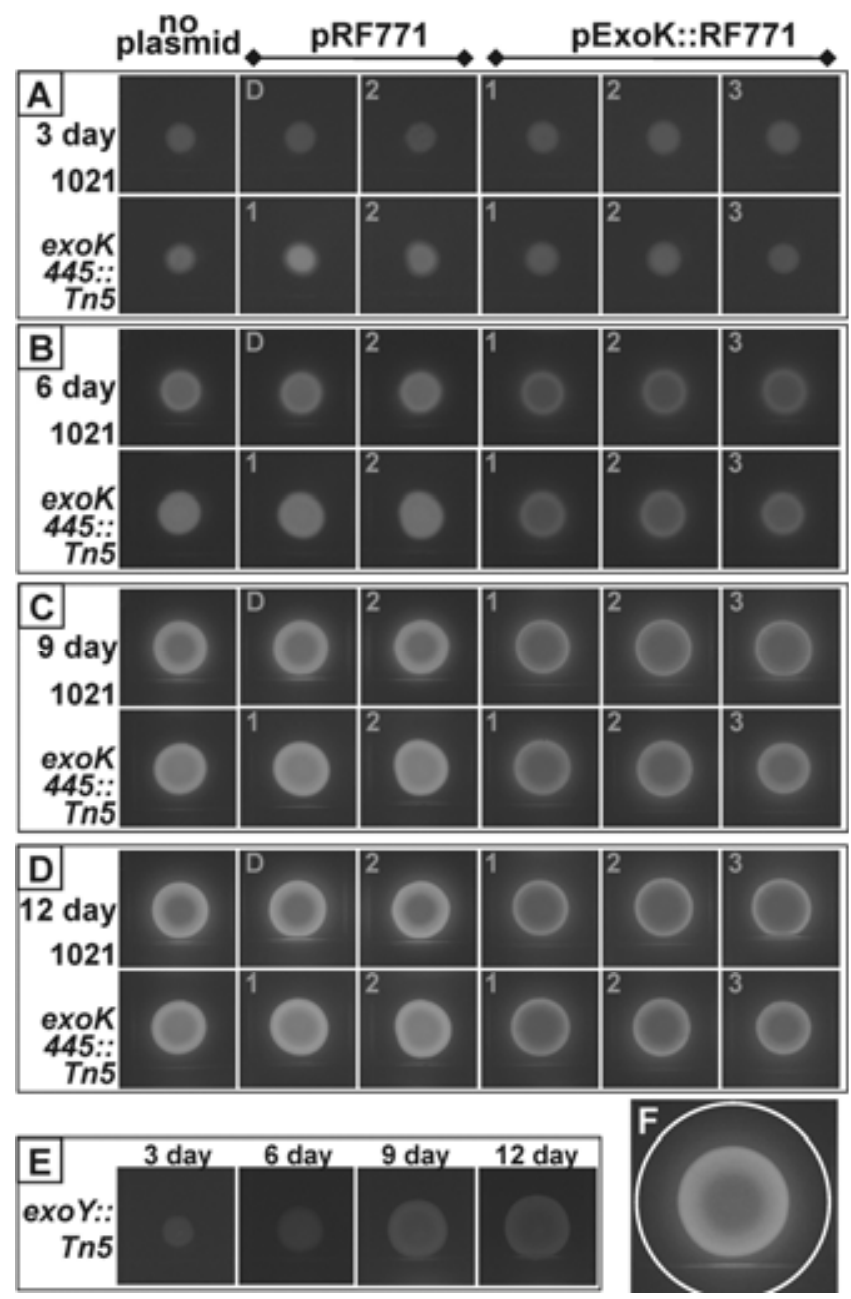

Fig. 4. Expression of exoK alone in pExoK::RF771 is sufficient to complement the delayed Calcofluor-halo phenotype of the exoK445::Tn5 mutant grown on LBMC (Luria-Bertani Miller medium supplemented with $\mathrm{MgSO}_{4}$ and $\mathrm{CaCl}_{2}$ ). After B, 6 and $\mathbf{C}, 9$ days, Sinorhizobium meliloti 1021 wild type (Sm1021[w.t.]) and Sm1021(w.t.) carrying the negative control construct pRF771 have a significant halo of Calcofluorstained LMW succinoglycan diffusing away from the spot of growing cells. Both Sm1021(w.t.) and exoK445::Tn5 carrying the pExoK::RF771 expression plasmid also have well-developed LMW halos at this time point. D, Uncomplemented exoK445::Tn5 mutant strains do not develop a significant halo until 12 days after inoculation, demonstrating the delayed and reduced production of LMW succinoglycan in these strains. E, For comparison, the same time points are shown for the Calcofluordark, succinoglycan-deficient exoY::Tn5 mutant. F, Magnified view of a single spot of growing $S$. meliloti cells with the margins of the Calcofluor halo outlined. All images were 0.25 -s exposures of 365-nm UV light. and exoK445::Tn5 have a more pronounced halo at 9 days' growth when they are overexpressing exoK from the pExoK::RF771 plasmid (Fig. 4C). This indicates that the reduction in LMW succinoglycan production in the exoK445::Tn5 mutant is complemented by the pExoK::RF771 plasmid, suggesting that the failure of this plasmid to complement the symbiotic defects of exoK445::Tn5 is not due to an absence of succinoglycan cleavage by ExoK.

\section{A secondary effect of the exoK445::Tn5 insertion is responsible for the lack of exoK complementation of the symbiotic defects of the exoK445::Tn5 mutant strains.}

We tested complementation by the pEX154 cosmid to confirm that the symbiotic defect of the exoK445::Tn5 strain is caused by a lesion in the same region of the $S$. meliloti genome that contains exoK (Fig. 5). The pEX154 cosmid carries an approximately $19.8-\mathrm{kb}$ fragment of $\mathrm{pSymB}$ DNA that extends from the exoT gene through the SMb20967 ORF (Long et al. 1988) (Figs. 1B and 6A). This cosmid has previously been demonstrated to complement the symbiotic defects of transposon insertion mutations in the exoH (Leigh et al. 1987), exoL, exoA, exoM, exoN, and exoP genes (Long et al. 1988). We found that this cosmid was able to complement the shoot growth (Fig. 5A) and nodule development (Fig. 5B) defects of the exoK445::Tn5 mutant, whereas the negative control construct pLAFR3 was not able to complement. (In sequencing the region surrounding the $\operatorname{Tn} 5$ insertion in the exoK445::Tn5 strain, we have not detected any additional mutations that could lead to complementation by pEX154 but not by exoK alone. This suggests that all of the defects in the exoK445::Tn5 strain are caused by the Tn 5 insertion itself.)

The most likely explanation for the complementation results described above is that insertion of the transposon in exoK445::Tn5 has a deleterious effect on expression of one or more of the downstream exo genes (exoLAMON) (Fig. 1B), and that this is responsible, in part, for the symbiotic phenotype. Schlüter and associates (2013) have reported a strong transcription start site 76 bp upstream of the annotated start site of exoH (pSymB position 1181162; transcript ID SmelB108) detected in an RNA pool that included $S$. meliloti grown in the presence of the plant flavonoid luteolin (Schluiter et al. 2013). This study also identified four additional, weaker transcription start sites within exoH and one within exoK (Schlüter et al. 2013). No transcription start sites were identified in the $495 \mathrm{bp}$ upstream of the exoL start codon (Schlüter et al. 2013), suggesting that most transcriptions of exoL (and likely the downstream exo genes as well) are dependent on read-through from the exoHK region.

To directly test the effect of the exoK445::Tn5 insertion on the expression of downstream genes, we performed quantitative reverse-transcriptase polymerase chain reaction (qRTPCR) on the exoL gene in Sm1021(w.t.) cells, the original exoK445::Tn5 mutant, and two exoK445::Tn5 strains generated by transduction of this insertion (Fig. 7). We found that exoL is expressed at low levels (compared with the trp promoter) in free-living cells grown in Luria-Bertani (LB) Miller medium supplemented with $2.5 \mathrm{mM} \mathrm{MgSO}_{4}$ and $2.5 \mathrm{mM}$ $\mathrm{CaCl}_{2}$ (LBMC) and that this level of expression is not dramatically altered in the exoK445::Tn5 mutant (Fig. 7A). We also tested the expression of exoL in these strains grown in Jensen's plant growth medium supplemented with $5 \mathrm{mM}$ glutamic acid sodium salt, $12.5 \mathrm{mM}$ mannitol, and biotin at 1 $\mu \mathrm{g} / \mathrm{ml}$ (JMG). Under these conditions, expression of exoL is extremely low and variable, and there are no significant differences in expression between Sm1021(w.t.) versus the exoK445::Tn5 strains (Fig. 7B). This is in agreement with the data of Becker and associates (1993), which showed that 
integration of the exoK disruption/reporter fusion plasmid pVXb1 did not prevent expression of the exoL gene by freeliving S. meliloti (Becker et al. 1993).

Although there do not appear to be any obvious effects of the exoK445::Tn5 insertion on the expression of downstream genes in the free-living state, it is possible that the exoL expression pattern in $S$. meliloti during symbiotic interaction with host plants is altered in the exoK445::Tn5 mutant strains. We have found that a $\beta$-glucuronidase (GUS)-reporter fusion to exoL (exoHKL::JH104) is expressed very strongly by $S$. meliloti in the $M$. truncatula nodule epidermis (Fig. 8A to D) at 10 days postinoculation and in nodules (Fig. $8 \mathrm{E}$ to $\mathrm{H}$ ) at 17 days postinoculation. These strains carry a duplication of a 3.4-kb region of pSymB extending from the $3^{\prime}$ end of SMb20952 to the $3^{\prime}$ end of exoL, and place the inserted GUS reporter under the same transcriptional controls as exoL. The transposon insertion in the exoK445::Tn5 mutant separates the exoLAMON genes from all but one of the transcription start sites identified by Schlüter and associates (2013), and polar effects of this insertion on symbiotic expression of these genes may explain the lack of complementation of the exoK445::Tn5 mutant by the exoK gene alone.

A nonpolar deletion of exoK has a small but significant loss of symbiotic productivity on both the host M. truncatula and the host alfalfa.

In order to dissect the effects of deletion and modification of the exoK and exoH genes on symbiosis and succinoglycan func-
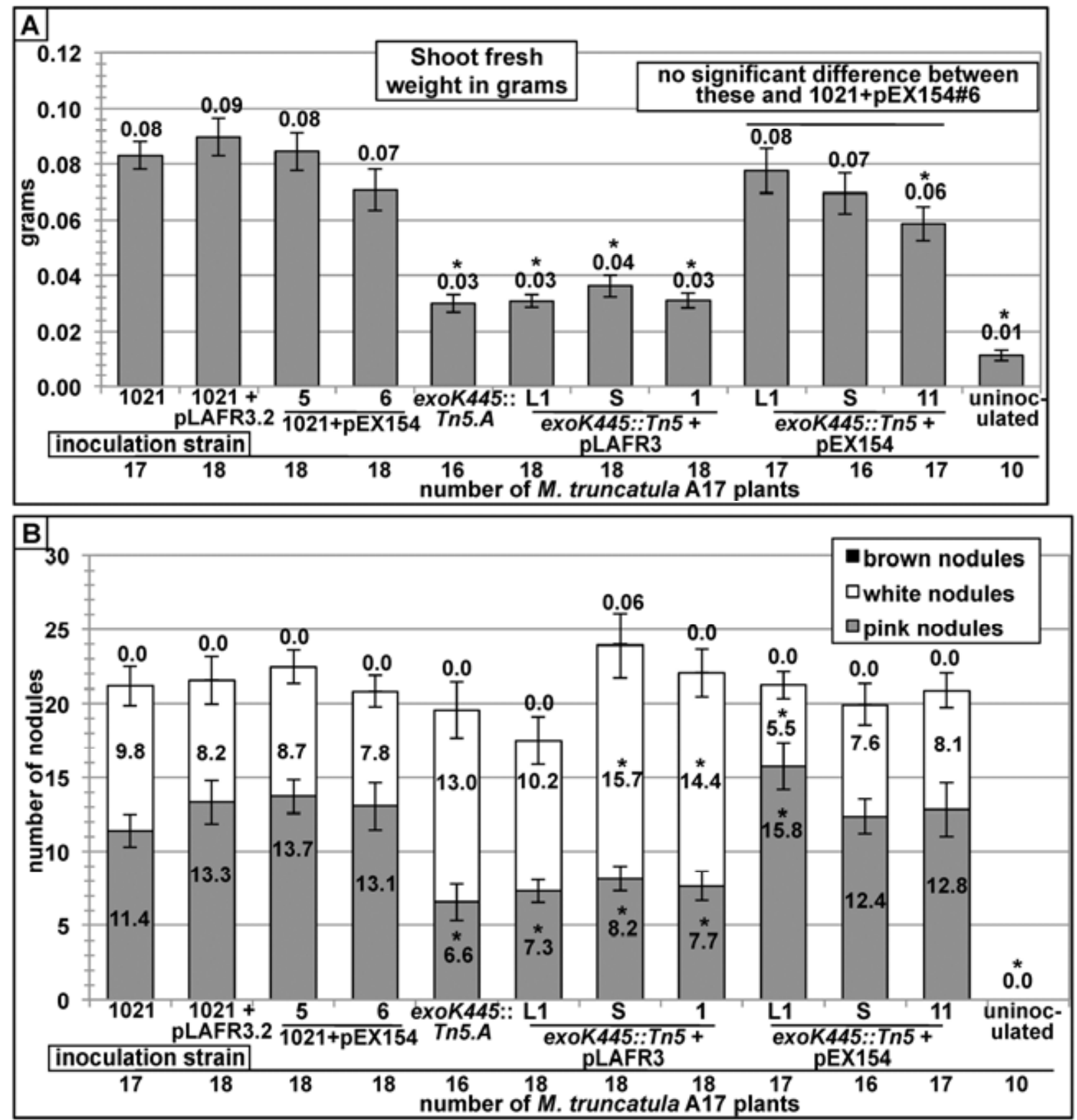

Fig. 5. Cosmid pEX154 complements the exoK445::Tn5 symbiotic defects in Medicago truncatula A17: A, shoot fresh weight and B, number of pink, functional nodules. The negative control construct pLAFR3 does not complement the defect. This is consistent with a secondary effect of the Tn5 insertion being at least partially responsible for the symbiotic growth defect of the exoK445::Tn5 mutant. Values for independently isolated strains of each genotype are grouped together (e.g., $1021+$ pEX154 "5" and "6"). Asterisk $(*)$ denotes statistically significant difference from plants inoculated with the Sinorhizobium meliloti 1021 wild-type reference strain. 
tion without deleterious polar effects on the exoLAMON operon, we constructed strains that place the exoLAMON genes under the control of a constitutive promoter and isolate this new artificial trp-exoLAMON operon from the exoHK genes. The goal was to generate strains in which the exoLAMON genes are under identical regulatory control despite alterations to the upstream exoHK region. The construction of these strains is shown in Figure 6B. The trpexoL strains are identical to Sm1021(w.t.) except that the trp promoter drives expression of the exoLAMON genes and a neomycin/kanamycin-resistance cassette $(\Omega N m / K m)$ separates these genes from exoHK (Fig. $6 \mathrm{~A}$ and $\mathrm{B})$. The Kdel-trpexoL strains are the same as the trpexoL strains except for the deletion of exoK (Fig. 6B). Expression of the exoL gene in i) LBMC medium and ii) JMG medium for two separately isolated trpexoL strains and three separately isolated Kdel-trpexoL strains is shown in Figure 7. The expression of exoL in these strains is $\geq 30$ fold more than that of Sm1021(w.t.) in LBMC medium, and $\geq 200$ fold more than that of Sm1021(w.t.) in JMG medium (Fig. 7). This demonstrates that we have produced strains that have strong, approximately equivalent expression of exoL regardless of the presence or absence of the exoK gene.

The symbiotic productivity (Fig. 9A) and nodule development (Fig. 9B) phenotypes of the trpexoL strains are not significantly different from those of Sm1021(w.t.) on the plant host $M$. truncatula. This demonstrates that there are no adverse effects on the symbiosis of placing the exoLAMON genes under the control of the trp promoter and placing the $\Omega N \mathrm{Nm} / \mathrm{Km}$ resistance cassette between the exoHK genes and the trp-exo$L A M O N$ operon. In contrast, the Kdel-trpexoL strains have a small but significant defect in both symbiotic productivity (Fig. 9A) and nodule development (Fig. 9B). The symbiotic defect of the Kdel-trpexoL strains on M. truncatula (shoot weight 60 to $70 \%$ that of $\mathrm{Sm} 1021$ [w.t.]) is not as severe as the defect of the exoK445::Tn5 strain (40\% that of Sm1021[w.t.]). This suggests that the exoK445::Tn5/M. truncatula symbiotic defects are due to the additive effect of the loss of exoK and a secondary effect of this transposon insertion. In the symbiosis with alfalfa, there is no significant difference in symbiotic productivity between the Kdel-trpexoL strains and the exoK445::Tn5 strain (Fig. 9C). Alfalfa plants inoculated with any of the exoK mutants have a shoot weight that is 54 to $69 \%$ that of Sm1021(w.t.) (Fig. 9C). This indicates that both plant hosts require exoK function for optimal symbiotic productivity. This also suggests that the difference in the extent of the symbiotic defect of the exoK445::Tn5 mutant on the two plant hosts is due to a secondary effect of the Tn5 insertion, which is more deleterious to the symbiosis with $M$. truncatula than alfalfa.

Unlike exoK445::Tn5, the defect in M. truncatula symbiotic productivity of the Kdel-trpexoL strains can be complemented by expression of exoK alone from the pExoK::RF771 plasmid (Fig. 10A), confirming that there are no secondary effects of the exoK deletion in the Kdel-trpexoL strains. This result is in striking contrast to the data in Figure $3 \mathrm{E}$ and $\mathrm{F}$, showing that the symbiotic defects of the exoK445::Tn5 mutant are not complemented by the pExoK::RF771 plasmid. These results also show that overexpression of exoK in Sm1021(w.t.) or in the trpexoL strains does not enhance symbiotic productivity with M. truncatula.

The Calcofluor-halo phenotype of the trpexoL strains is similar to that of the Sm1021(w.t.) strain when these strains are grown on LBMC medium (Fig. 11A). Compared with these strains, at 6 and 9 days of growth, the Kdel-trpexoL strains have a delay in halo formation similar to that of the exoK445::Tn5 strains (Fig. 11A). In JMG plant medium, the delay in halo formation of the exoK445::Tn5 strains and the Kdel-trpexoL strains relative to $\mathrm{Sm} 1021$ (w.t.) and the trpexoL strains is less obvious but it can be observed at 6 days of growth (Fig. 11B). This indicates that the loss of exoK has a similar effect on the production of LMW succinoglycan in the Kdel-trpexoL strains and in the exoK445::Tn5 strains.

\section{DISCUSSION}

We have shown that the loss of the exoK glycanase in $S$. meliloti 1021 results in reduced symbiotic efficiency on both $M$. truncatula and alfalfa. We have also shown that the exoK445::Tn5 mutant strain has a more pronounced symbiotic defect on $M$. truncatula than on alfalfa, whereas the nonpolar Kdel deletion strains have a similar reduction in symbiotic productivity on both of these hosts. This indicates that the reduction in the amount of LMW succinoglycan due to the loss of exoK leads to a similar loss in symbiotic efficiency on both hosts, and that it is a secondary effect of the Tn5 insertion in the exoK445::Tn5 strain that leads to the more pronounced symbiotic defect with $M$. truncatula. This secondary effect can be complemented by the pEX154 cosmid, which carries the pSymB gene region from exoT to SMb20967 (Fig. 1B). This is consistent with the secondary effect of the Tn 5 insertion producing a polar effect on the downstream exoLAMON genes that is not apparent in the free-living state but causes a defect in the symbiotic interaction with M. truncatula. This is also consistent

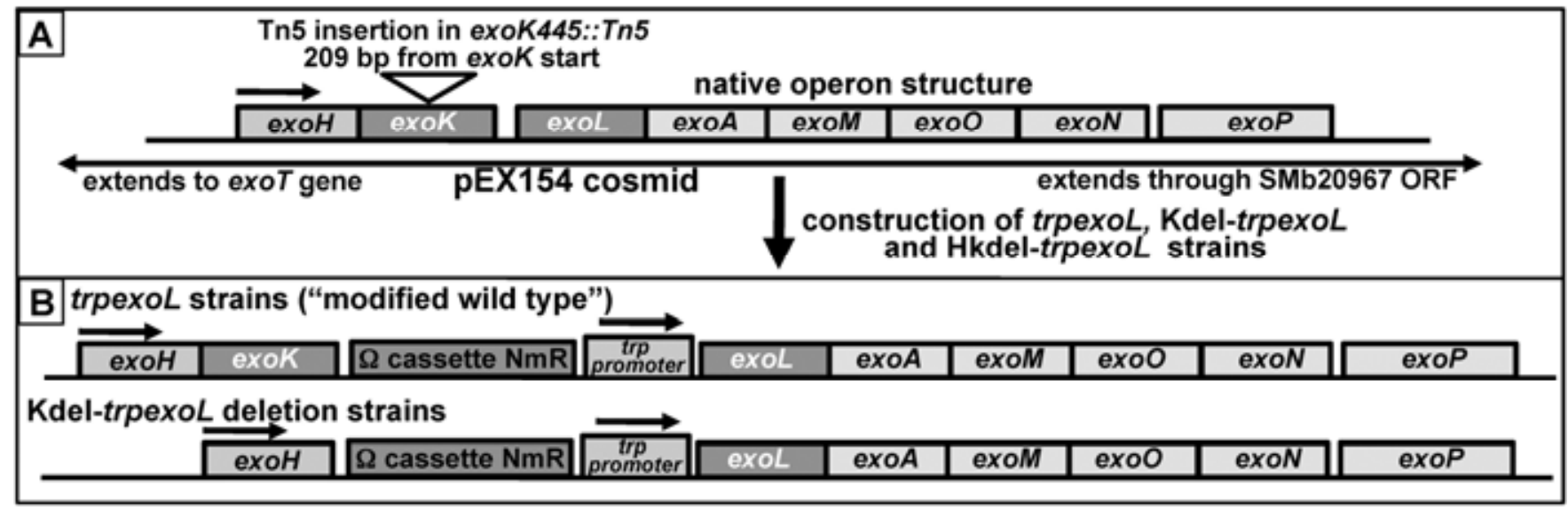

Fig. 6. A, Arrangement of the exoHKLAMON genes on the pSymB plasmid of Sinorhizobium meliloti 1021 . The extent of the region carried on the pEX154 cosmid is also shown. B, Construction of the trpexoL and Kdel-trpexoL strains in order to eliminate polar effects on the exoLAMON genes. In all of these strains, the exoLAMON genes are isolated from the upstream exoHK genes by an $\Omega \mathrm{Nm}^{\mathrm{r}}$ cassette carrying neomycin and kanamycin resistance. In these strains, the trp promoter drives the expression of exoLAMON. 
with results from Jones (2012) demonstrating that increasing succinoglycan production increases the efficiency of the symbiosis with $M$. truncatula but has no effect on the symbiosis with alfalfa (Jones 2012). Perhaps any perturbation of succinoglycan production due to negative effects on exoLAMON expression pushes the succinoglycan level below a critical threshold level for successful symbiosis with $M$. truncatula.

We have also shown that there is strong expression of exoL by $S$. meliloti during invasion of $M$. truncatula and in nodules, despite weak expression in Jensen's medium. Succinoglycan production has been shown to be increased by high phosphate concentration (Mendrygal and González 2000) and it is possible that phosphate is involved in regulation of exoHKLAMON expression on plants, because phosphate levels are usually higher on plant roots (Bieleski 1973; Israel 1987) than the 8.5 $\mathrm{mM}$ found in Jensen's medium (Jones 2012; Mendrygal and González 2000). It is also possible that other compounds produced by host plants, such as flavonoids (Peck et al. 2006), affect expression of the exoHKLAMON genes.
In another rhizobial symbiont-host plant pair, there is an apparent contrast to our current results. An exoK $\Omega$-cassette insertion mutant of $S$. fredii NGR234 produces no pink, functional nodules on the host plant L. leucocephala (Staehelin et al. 2006). However, complementation of this mutant with a plasmid carrying the NGR234 exoK gene produces an intermediate phenotype: $3.1 \pm 0.8$ pink nodules were produced per plant relative to the $10.3 \pm 1.5$ pink nodules/plant produced by plants inoculated with the $S$. fredii NGR234 wild-type strain carrying the exoK plasmid (Staehelin et al. 2006). In S. fredii NGR234, the order of the exoKLAMON genes is the same as in S. meliloti 1021 (Schmeisser et al. 2009). One possibility is that the intermediate phenotype of the NGR234 exoK $\Omega$-cassette insertion + pExoK strain is due a lack of complementation by exoK of a secondary effect of the insertion, analogous to the lack of complementation of the $S$. meliloti exoK445::Tn5 symbiotic phenotype by expression of exoK in $S$. meliloti.

There are several critical questions for future study. For example, does the intermediate symbiotic phenotype caused by
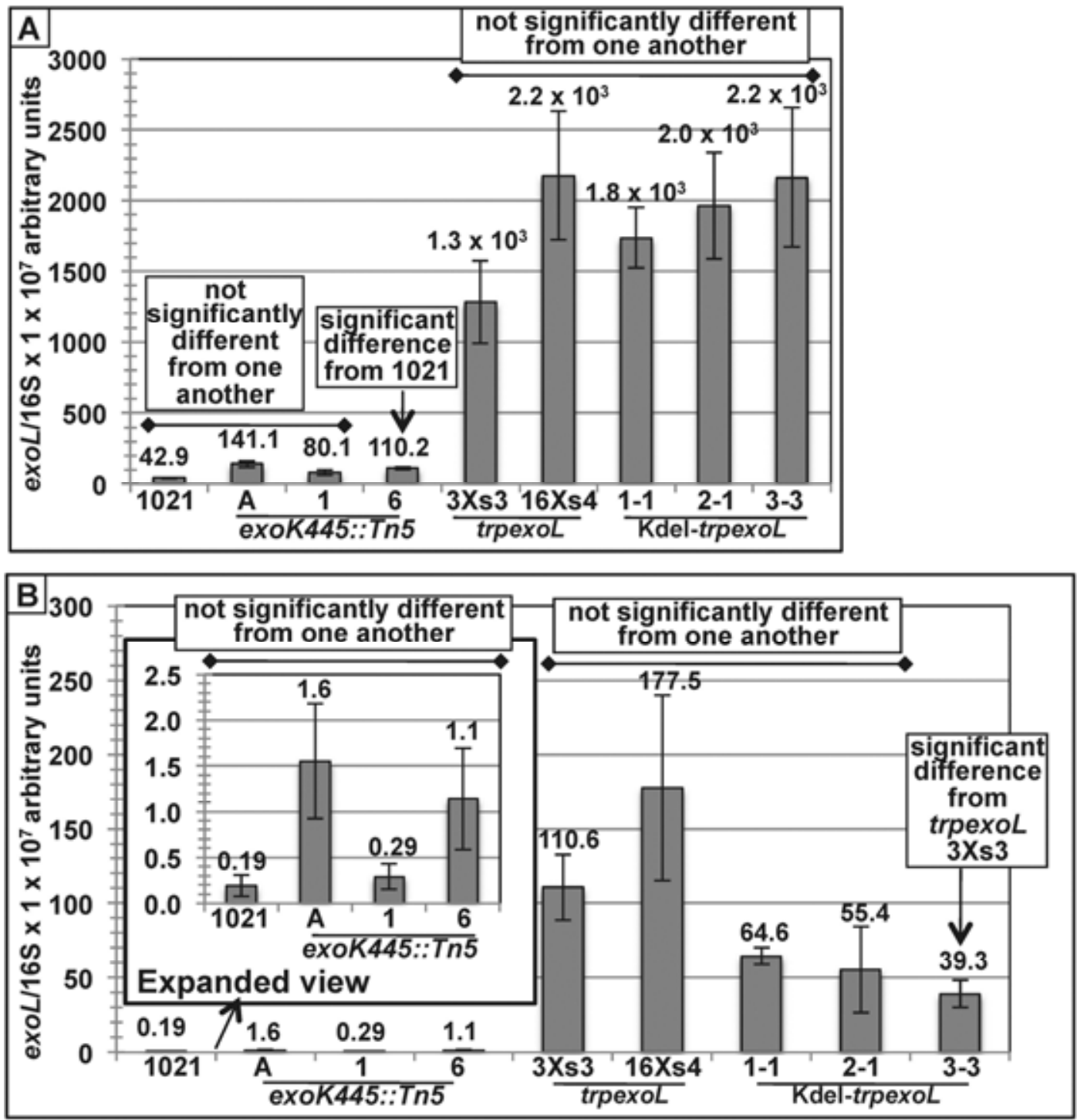

Fig. 7. Expression of the exoL gene in A, LBMC (Luria-Bertani Miller medium supplemented with $\mathrm{MgSO}_{4}$ and $\mathrm{CaCl}_{2}$ ) and $\mathbf{B}$, JMG (Jensen's plant growth medium supplemented with glutamic acid sodium salt, mannitol, and biotin) relative to $16 \mathrm{~S}$ rRNA expression (measured by quantitative reverse-transcriptase polymerase chain reaction and expressed in arbitrary units multiplied by $10^{7}$ ). In both LBMC and JMG, exoL expression under the control of the trp promoter is much stronger than expression from the native promoter in Sinorhizobium meliloti 1021 wild type (Sm1021[w.t.]). Free-living bacteria in both LBMC and JMG have low-level expression of exoL in Sm1021(w.t.) and in the exoK445::Tn5 strains. Inset in B is an adjusted-scale view of exoL expression in 1021 and exoK::Tn5 1, 1.5, and 6.2. The only significant difference in exoL expression from Sm1021(w.t.) by an exoK445::Tn5 strain is in exoK::Tn5 6.2 in LBMC medium. The only significant difference in exoL expression among the trpexoL and Kdel-trpexoL strains is in Kdel-trpexoL.3.3 in JMG medium (B). 

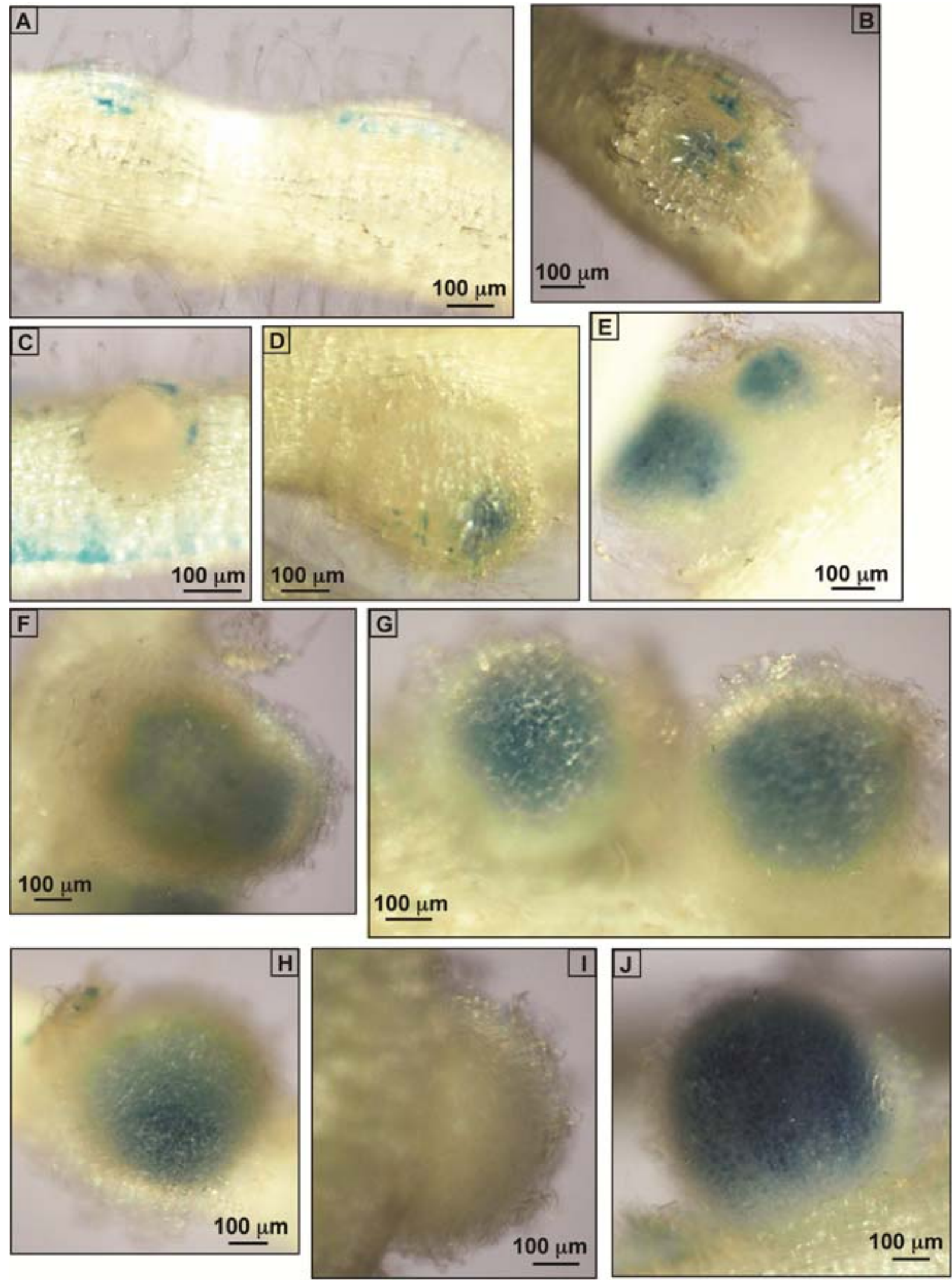

Fig. 8. A to D, Expression of an exoHKL:: $\beta$-glucuronidase (GUS) reporter fusion on the Medicago truncatula developing nodule epidermis at 10 days postinoculation and $\mathbf{E}$ to $\mathbf{H}$, in nodules at 17 days postinoculation. The GUS reporter in the inserted plasmid pJH104 is under the transcriptional control of a duplicated 3.4-kb region of pSymB extending from the $3^{\prime}$ end of SMb20952 to the $3^{\prime}$ end of exoL. Five independently isolated fusion strains are shown: A, exoHKL::JH104.1C14; $\mathbf{B}$ and $\mathbf{E}$, exoHKL::JH104.2C10; C and D, exoHKL::JH104.2B6; F, exoHKL::JH104.1B6; G and H, exoHKL::JH104.2C12. I, Negative control, S. meliloti 1021 without a GUS fusion (17 days postinoculation). J, Positive control for strong GUS expression from the SMc00911::JH104 reporter fusion (Queiroux et al. 2012) (17 days postinoculation). In all panels, the bar corresponds to $100 \mu \mathrm{m}$. Roots were stained for 23 to $24 \mathrm{~h}$ (A to $\mathrm{C}$ and $\mathrm{E}$ to $\mathrm{J}$ ) or $47 \mathrm{~h}(\mathrm{D})$. 

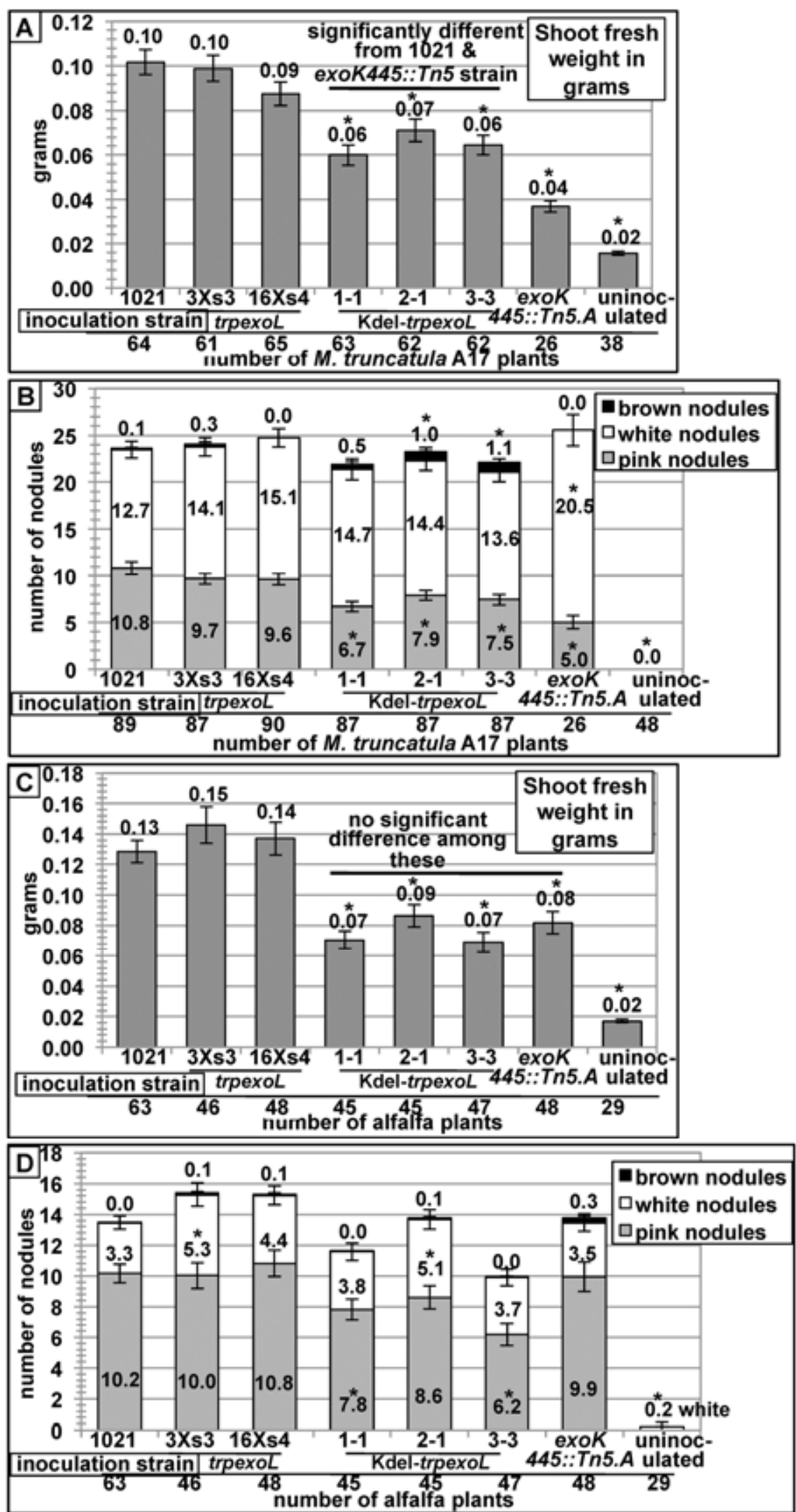

Fig. 9. Symbiotic performance of Medicago truncatula plants inoculated with two independently isolated trpexoL strains is not significantly different from those inoculated with the Sinorhizobium meliloti 1021 wild type (Sm1021[w.t.]), measured by A, shoot fresh weight or B, number of pink, functional nodules. In contrast, the Kdel-trpexoL strains carrying a deletion of the exoK gene have a significantly reduced $\mathbf{A}$, shoot weight and $\mathbf{B}$, number of pink, functional nodules relative to those inoculated with the S. meliloti Sm1021(w.t.). However on M. truncatula, the symbiotic defects of the Kdel-trpexoL strains are not as severe as those of the exoK445::Tn5 mutant. On the plant host alfalfa, the $\mathbf{C}$, symbiotic productivity and $\mathbf{D}$, nodule development of the Kdel-trpexoL and exoK445::Tn5 strains are not significantly different from one another. Values for independently isolated strains of each genotype are grouped together (e.g., trpexoL "3Xs3" and "16Xs4"). Asterisk (*) denotes statistically significant difference from plants inoculated with the $S$. meliloti Sm1021(w.t.) reference strain. Data are averaged from five separate experiments. 
loss of exoK in the $S$. meliloti Kdel-trpexoL strains mean that LMW succinoglycan is beneficial but not required for host invasion, or are there alternate mechanisms for producing LMW succinoglycan during host invasion? It is known that the glycanase encoded by exs $H$ cleaves succinoglycan in vitro and that it contributes to the production of LMW succinoglycan by S. meliloti in the free-living state (York and Walker 1997, 1998a). However, it is not clear that the exs $H$-encoded glycanase makes any contribution to the host invasion efficiency of $S$. meliloti. A double mutant in exoK445::Tn5/exsH was found to be capable of symbiosis with the host plant alfalfa but the symbiotic phenotypes were not quantified (York and Walker 1997). It may be that another succinoglycan glycanase contributes to the production of LMW succinoglycan during host invasion or that the LMW fraction of succinoglycan is beneficial but not required for host invasion. We are currently determining the molecular weight distribution of succinoglycan produced by the Kdel-trpexoL/exsH double mutants under free-living conditions that mimic as closely as possible those the bacteria en- counter on host plants to determine whether any LMW succinoglycan can be detected in these strains. We are also determining whether other putative glycanases encoded in the $S$. meliloti 1021 genome can contribute to the production of LMW succinoglycan, and whether these glycanases can complement the reduced symbiotic efficiency of the Kdel-trpexoL mutant strains.

Acidic rhizobial EPS are required for host invasion or contribute to host invasion efficiency in many different symbionthost pairs (Borthakur et al. 1986; Diebold and Noel 1989; Kelly et al. 2013; Laus et al. 2005; Staehelin et al. 2006; van Workum et al. 1995). A role for these rhizobial EPS in induction of infection thread formation via a signaling pathway is possible; however, it is also possible that these compounds act by altering the conditions within the infection thread. Acidic EPS produced by plant-pathogenic bacteria are also important determinants of virulence in plant disease. For example, in Xanthomonas campestris pv. campestris, production of the polysaccharide xanthan enhances bacterial infection and tissue damage of host plants (Newman et al. 1994; Yun et al. 2006).
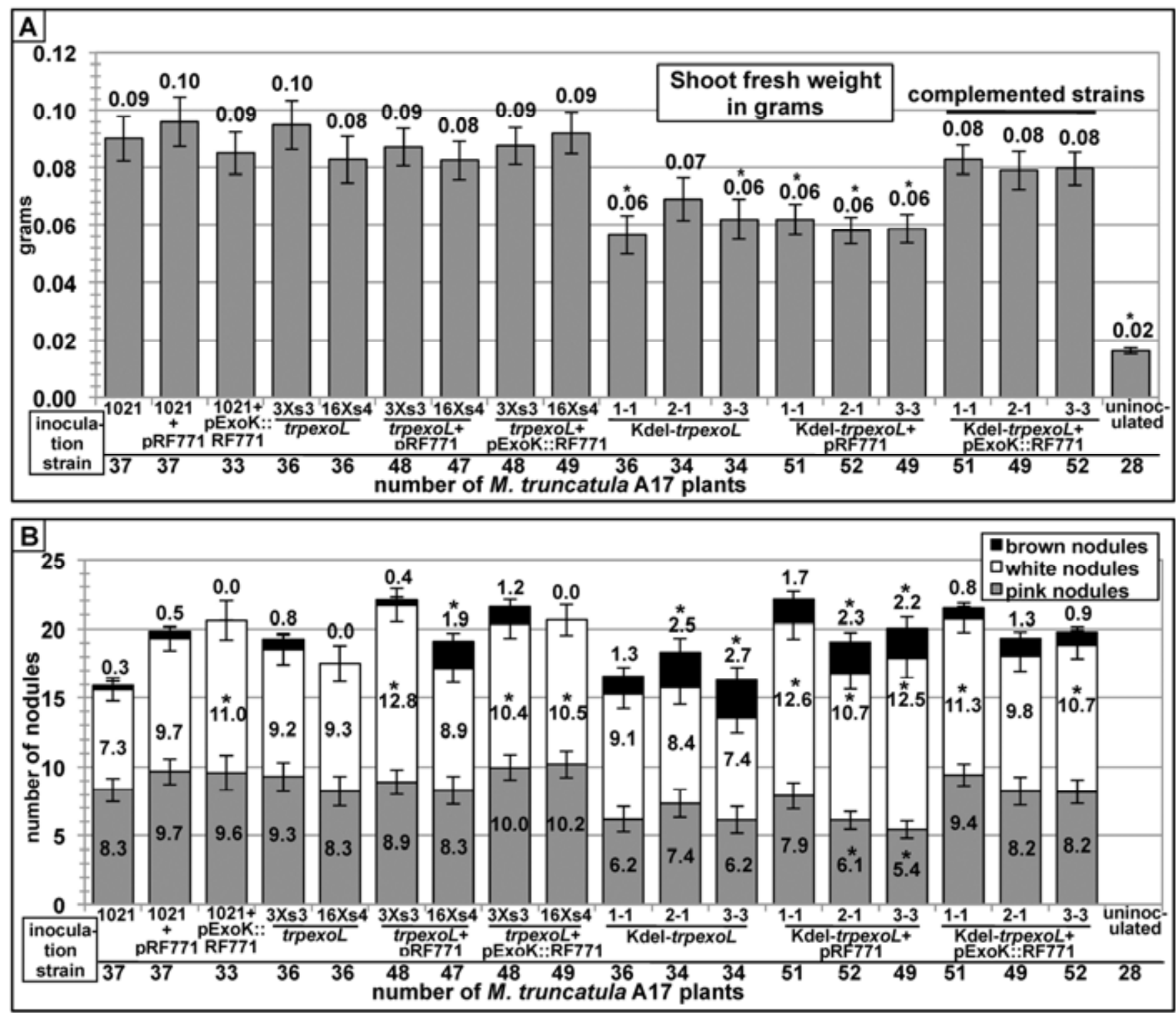

Fig. 10. Medicago truncatula symbiotic defects of the Kdel-trpexoL strains are complemented by expression of exoK alone. A, Shoot fresh weight and $\mathbf{B}$, number of pink, functional nodules of plants inoculated with the complemented strains are not significantly different from those inoculated with Sinorhizobium meliloti 1021 wild type (Sm1021[w.t.]) or the trpexoL strains. Values for independently isolated strains of each genotype are grouped together (e.g., trpexoL "3Xs3" and "16Xs4"). Asterisk (*) denotes statistically significant difference from plants inoculated with the S. meliloti Sm1021(w.t.) reference strain. Data are averaged from three separate experiments. 
Xanthan and other bacterial polysaccharides have been demonstrated to suppress plant defense responses by binding $\mathrm{Ca}^{2+}$ and preventing the activation of these responses by $\mathrm{Ca}^{2+}$-signaling (Aslam et al. 2008). Also, the acidic EPS alginate exacerbates disease symptoms caused by Pseudomonas syringae on host plants (Yu et al. 1999). It has been proposed that both alginate and xanthan aid in host infection by absorbing water and creating a hydrated microenvironment around the invading bacteria (Beattie 2011; Yu et al. 1999). It is possible that bacterial acidic EPS facilitate these very different symbiotic and pathogenic bacteria-host interactions by performing similar chemical or mechanical functions. During infection thread formation by symbiotic rhizobia, acidic EPS might be involved in modulating $\mathrm{Ca}^{2+}$ levels or in hydration of the infection thread matrix. Cleavage of EPS into smaller LMW units would be expected to raise the osmolarity (Buchanan et al. 2000) and, thus, the osmotic potential of the solution in the expanding portion of the infection thread, which might facilitate infection thread extension.

We have determined that $S$. meliloti 1021 strains in which the loss of exoK is the sole genetic defect have reduced symbiotic productivity on both the plant hosts $M$. truncatula and alfalfa. The exoK glycanase is known to contribute to the production of LMW succinoglycan under free-living conditions. These results suggest that LMW succinoglycan is beneficial but not absolutely required for this rhizobial species to establish a symbiosis with its host plants, or that exs $H$ or another one of the putative glycanases being investigated can partially replace the function of exoK during symbiosis.

\section{MATERIALS AND METHODS}

Bacterial strains and growth conditions.

S. meliloti 1021 strains (Supplementary Table 1) (Meade et al. 1982) were grown at $30^{\circ} \mathrm{C}$ in either LBMC medium or JMG (Jones 2012; Leigh et al. 1985; Vincent 1970). Bacterial plates contained 1.5\% Bacto agar (BD, Franklin Lakes, NJ, U.S.A.). Calcofluor polysaccharide indicator plates contained $0.02 \%$ Calcofluor white M2R (fluorescent brightener 28; Sigma-Aldrich, St. Louis) (Leigh et al. 1985). S. meliloti 1021 was grown with streptomycin at $500 \mu \mathrm{g} / \mathrm{ml}$. S. meliloti strains carrying pLAFR-based cosmids and pRF771-based plasmids were also grown with tetracycline at $10 \mu \mathrm{g} / \mathrm{ml}$. S. meliloti strains carrying pMSO4-based plasmids were also grown with spectinomycin at $100 \mu \mathrm{g} / \mathrm{ml}$. S. meliloti strains carrying transposon $\mathrm{Tn} 5$, the $\mathrm{Nm} / \mathrm{Km} \Omega$-cassette, or pJH104 insertions were also grown with neomycin at $200 \mu \mathrm{g} / \mathrm{ml}$. Escherichia coli strains were grown at $37^{\circ} \mathrm{C}$ in LB medium (Sambrook et al. 1982), with appropriate antibiotics used at the following concentrations: kanamycin at $50 \mu \mathrm{g} / \mathrm{ml}$, chloramphenicol at $10 \mu \mathrm{g} / \mathrm{ml}$, tetracycline at $10 \mu \mathrm{g} / \mathrm{ml}$, and spectinomycin at $100 \mu \mathrm{g} / \mathrm{ml}$.

\section{Construction of $S$. meliloti mutant strains.}

All restriction enzymes and Phusion High-Fidelity polymerase were obtained from New England Biolabs (Ipswich, MA, U.S.A.). All primers were obtained from Eurofins MWG Operon, (Huntsville, AL, U.S.A.). Plasmids pExoK::MSO4 and pExo::RF771 that constitutively express exoK were constructed by amplifying the exoK open reading frame (ORF) with primers

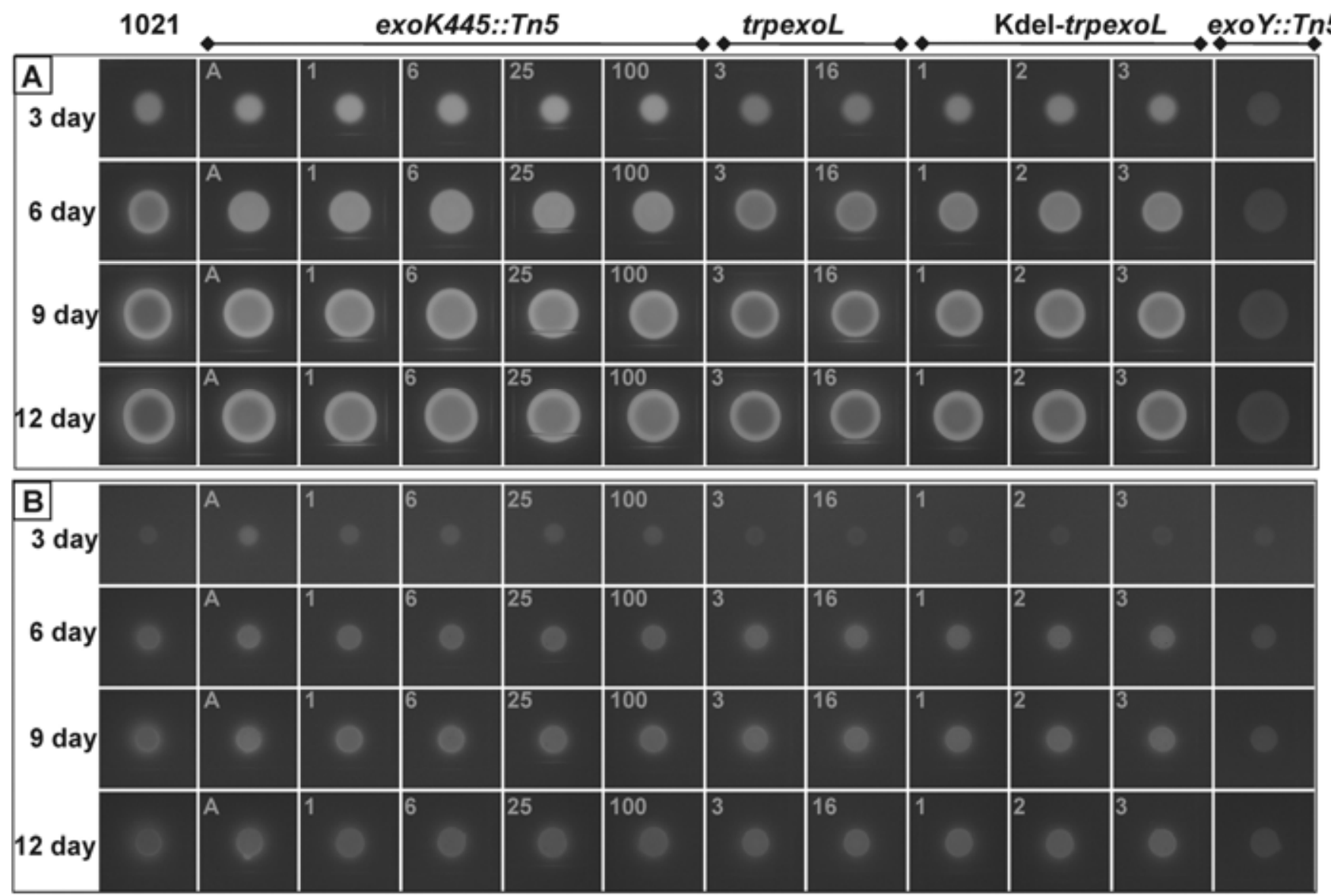

Fig. 11. A, Kdel-trpexoL strain has a delayed Calcofluor-halo phenotype similar to those of the exoK445::Tn5 strains on LBMC (Luria-Bertani Miller medium supplemented with $\mathrm{MgSO}_{4}$ and $\mathrm{CaCl}_{2}$ ). The delay in halo formation by these strains relative to Sinorhizobium meliloti 1021 wild type (Sm1021[w.t.]) and the trpexoL strains is most obvious after 9 days of growth. B, On Jensen's medium, the difference in halo formation is less apparent but, at both 6 and 9 days of growth, the exoK445::Tn5 and Kdel-trpexoL strains have a fainter halo than Sm1021(w.t.) and the trpexoL strains. All exposures were 0.25 s with 365-nm UV light illumination. 
exoK.rbs.fwd.Pst and exoK.rev.BamHI, using Phusion polymerase, and cloning into pCR-Blunt II-TOPO using the ZeroTOPO-Blunt cloning kit (Life Technologies, Carlsbad, CA, U.S.A.), creating plasmid exoK.Pst/Bam/TOPO.1. The ribosome binding site used for translation of exoK (Gage et al. 1996) was incorporated into the exoK.rbs.fwd.Pst primer. The exoK ORF PstI/BamHI fragment from exoK.Pst/Bam/TOPO.1 was cloned into plasmid pMSO4 (Barnett et al. 2000) to make plasmid pExoK::MSO4.PB, placing exoK under the control of the trp promoter, which is constitutively active in $S$. meliloti (Egelhoff and Long 1985). This fragment was also cloned into plasmid pRF771 (Wells and Long 2002) to make plasmid pExoK::RF771. Plasmids were mobilized into $S$. meliloti by triparental conjugation, as described (Backman and Boyer 1983; Finan et al. 1986; Glazebrook and Walker 1991). S. meliloti exconjugants carrying pMSO4 or pExoK::MSO4.PB were selected on LBMC medium containing spectinomycin at $100 \mu \mathrm{g} / \mathrm{ml}$ and streptomycin at $1,000 \mu \mathrm{g} / \mathrm{ml}$. Exconjugants carrying pRF771, pExoK::RF771, pLAFR3 (Staskawicz et al. 1987), or pEX154 (Leigh et al. 1987) were selected on LBMC medium containing tetracycline at $10 \mu \mathrm{g} / \mathrm{ml}$ and streptomycin at $1,000 \mu \mathrm{g} / \mathrm{ml}$.

S. meliloti 1021 exoHKL::JH104 GUS-reporter insertion strains were constructed by amplifying a $3.4-\mathrm{kb}$ product from within the 3' end of SM_b20952 to the $3^{\prime}$ end of exoL using primers exoH5'gus.rep.fwd and exoL3'gus.rep.rev and cloning the amplified product into the plasmid pJH104 (Ferguson et al. 2005). The resulting plasmid, pexoHKL::JH104, was conjugated into $S$. meliloti 1021 and exconjugants were selected on LBMC medium containing neomycin at $200 \mu \mathrm{g} / \mathrm{ml}$ and streptomycin at $1,000 \mu \mathrm{g} / \mathrm{ml}$. The exconjugants exoHKL::JH104.1B6, exoHKL:: JH104.1C14, exoHKL::JH104.2B6, exoHKL::JH104.2C10, and exoHKL::JH104.2C12 were confirmed to have a complete duplication of the region from the $3^{\prime}$ end of SM_b20952 to the $3^{\prime}$ end of exoL, generating a GUS reporter fusion to exoL.

$S$. meliloti strains trpexoL3Xs3 and trpexoL16Xs4, in which the exoLAMON operon is expressed under the control of the trp promoter and is separated from the exoHK genes by a

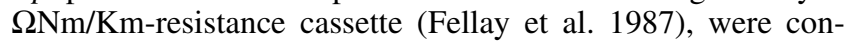
structed using the plasmid pK3.trpexoKm2. This plasmid was constructed as follows. The trp promoter was amplified from pMSO4 (Barnett et al. 2000) and was fused with a 0.97-kb fragment of exoL (beginning with the start codon) using primers trp/exoL.up1.SalI, trp/exoL.dwn1, trp/exoL.up2 and trp/ exoL. dwn2.XhoI. The fusion primers trp/exoL.dwn1 and trp/exoL.up2 also introduced a ribosome-binding site and spacer between the trp promoter and the exoL start codon. This fragment was cloned into the SalI and XhoI sites of the $s a c B$ gene-containing suicide plasmid pJQ100SK (Quandt and Hynes 1993). The resulting plasmid was then digested with BamHI and ligated with the $B a m \mathrm{HI} \mathrm{Nm} / \mathrm{Km}$-resistance cassette fragment from $\mathrm{pHP} 45 \Omega-\mathrm{Nm} / \mathrm{Km}$ (Fellay et al. 1987) to generate plasmid ptexoL- $\Omega \mathrm{Km}$. This plasmid was digested with $\mathrm{SacI}$ and $\mathrm{XbaI}$ and ligated with a $0.8-\mathrm{kb} S a c \mathrm{I} / \mathrm{XbaI}$ fragment that includes the $3^{\prime}$ end of the exoK gene (amplified with primers exoK.fwd.SacI and exoK.rev.XbaI) to generate plasmid pK3.trpexoKm2. This plasmid was conjugated into S. meliloti 1021 to generate strains in which the $\mathrm{Nm} / \mathrm{Km}$-resistance cassette has been inserted immediately $3^{\prime}$ of exoK, followed by the trp promoter driving expression of the exoLAMON operon (Fig. 6, diagram). Insertion-carrying exconjugants were selected on neomycin at 200 $\mu \mathrm{g} / \mathrm{ml}$ and streptomycin at $1,000 \mu \mathrm{g} / \mathrm{ml}$. Double-recombinant gene-replacement strains trpexoL3 and trpexoL16 were selected for the loss of the $s a c B$ gene carried by pJQ100SK on $1 / 10$ LBMC $+7 \%$ sucrose medium, with neomycin at 200 $\mu \mathrm{g} / \mathrm{ml}$ and streptomycin at $1,000 \mu \mathrm{g} / \mathrm{ml}$. The $\mathrm{Nm} / \mathrm{Km}-$ resistance markers from trpexoL3 and trpexoL16 were transduced into S. meliloti 1021 using the phage $\phi$ M12 (Finan et al.
1984) to generate strains trpexoL3Xs3 and trpexoL16Xs4. The presence of the $\mathrm{Nm} / \mathrm{Km}$-resistance cassette and the trp promoter between exoK and exoL was confirmed by PCR.

The strains Kdel-trpexoL.1-1, Kdel-trpexoL.2-1, and KdeltrpexoL.3-3 were constructed using the plasmid Kdelp.texoL. This plasmid was constructed from plasmid ptexoL- $\Omega \mathrm{Km}$ (discussed above). Plasmid ptexoL- $\Omega \mathrm{Km}$ was digested with Eco53kI and $X b a \mathrm{I}$ and was ligated with a $0.9-\mathrm{kb} E c o \mathrm{RV} / X b a \mathrm{I}$ fragment that includes the sequence immediately $5^{\prime}$ of the exoK gene (amplified with primers Kdel.trpexo1fwd and Kdel.trpexo1rev). This generated plasmid Kdelp.texoL, which was conjugated into $S$. meliloti 1021 to generate strains KdeltrpexoL.1, Kdel-trpexoL.2, and Kdel-trpexoL.3, in which the $\mathrm{Nm} / \mathrm{Km}$-resistance cassette has been inserted immediately $5^{\prime}$ of exoK, deleting exoK, and followed by the trp promoter driving expression of the exoLAMON operon (Fig. 6, diagram). Exconjugants were selected as described above for strains trpexoL3 and trpexoL16. The $\mathrm{Nm} / \mathrm{Km}$-resistance markers from strains Kdel-trpexoL.1, Kdel-trpexoL.2, and Kdel-trpexoL.3 were $\phi M 12$-transduced into S. meliloti 1021 to generate strains Kdel-trpexoL.1-1, Kdel-trpexoL.2-1, and Kdel-trpexoL.3-3.

\section{Plant nodulation assays.}

The host plant Medicago sativa (alfalfa) 'Iroquois' was prepared for inoculation with $S$. meliloti as described by Leigh and associates (1985) with modifications: seed were sterilized for $5 \mathrm{~min}$ in $50 \%$ bleach, rinsed in sterile water, and germinated for 3 days on plant cell culture-tested $1.1 \%$ (wt/vol) agar-water (Sigma-Aldrich). Seedlings were then moved to individual 100-by-15-mm Jensen's medium microcosm plates (Vincent 1970) and inoculated with $100 \mu \mathrm{l}$ of $S$. meliloti of the appropriate strain at an optical density of 0.05 at wavelength $600 \mathrm{~nm}$. Plants were grown in a Percival AR-36L incubator (Perry, IA, U.S.A.) at $21^{\circ} \mathrm{C}$ with 60 to $70 \%$ relative humidity and light at 100 to $175 \mu \mathrm{mol} \mathrm{m} \mathrm{m}^{-2} \mathrm{~s}^{-1}$ for 5 weeks. The host plant M. truncatula (barrel medic) Jemalong A17 was prepared for inoculation with $S$. meliloti by scarifying seed in concentrated sulfuric acid for 10 to $12 \mathrm{~min}$, rinsing eight times in sterile water, soaking overnight at $4^{\circ} \mathrm{C}$, and germinating for 3 days on plant cell culture-tested $1.1 \%$ (wt/vol) agar-water (Penmetsa and Cook 2000). Seedlings were then treated, inoculated, and maintained as for alfalfa (discussed above) for 7 weeks.

\section{Western blotting.}

Each stationary phase culture $(20 \mu \mathrm{l})$ grown in MGS medium (York and Walker 1997) was added to $105 \mu \mathrm{l}$ of sodium dodecyl sulfate (SDS) lysis buffer (final concentration 5\% $\beta$-mercaptoethanol, 2.3\% SDS, 8\% glycerol, $42 \mathrm{mM}$ Tris [pH 6.8], and $0.005 \%$ Bromphenol blue) and boiled for $5 \mathrm{~min}$. Each cleared lysate $(12 \mu \mathrm{l})$ was loaded onto a $12 \%$ SDS polyacrylamide gel electrophoresis gel (Bio-Rad mini-PROTEAN gel apparatus; Bio-Rad, Hercules, CA, U.S.A.). Approximately equivalent quantities of total protein were loaded, as measured by Bradford assay (Bradford, 1976). The gel was then blotted to polyvinylidene diflouride for $1 \mathrm{~h}$ in Tris/glycine/methanol buffer according to the manufacturer's recommendations (BioRad mini Trans-Blot). The blot was blocked with 3\% gelatin and incubated with $\alpha$-exoK antisera (provided by G. Walker, Massachusetts Institute of Technology) (York and Walker 1998a) at a 1:400 dilution for $3 \mathrm{~h}$ at room temperature. Anti-rabbit immunoglobulin $\mathrm{G}$ secondary antibody and alkaline phosphatase substrate were used according to the manufacturer's suggestions (Bio-Rad Immun-blot AP colorometric kit).

\section{qRT-PCR assays.}

RNA was isolated from 1.2 to $1.25 \mathrm{ml}$ of culture from triplicate samples of each $S$. meliloti strain grown in either LBMC 
or JMG medium. RNA was isolated using the Ambion Totally RNA kit (Life Technologies, Carlsbad, CA, U.S.A.). RNA was treated with DNase I from the Zymo DNA-free clean-up kit (Zymo Research, Irvine, CA, U.S.A.), and test qPCR tests performed to ensure that no DNA remained. Total RNA $(225 \mu \mathrm{g})$ was reverse transcribed with random hexamers using Superscript III, followed by RNase $\mathrm{H}$ treatment (Life Technologies). qPCR of exoL was performed in a Bio-Rad CFX96 real-time cycler (conditions: $98^{\circ} \mathrm{C}$ for $2 \mathrm{~min} ; 39$ cycles of $98^{\circ} \mathrm{C}$ for $5 \mathrm{~s}$ and $65^{\circ} \mathrm{C}$ for $5 \mathrm{~s}$; and melt curve of 65 to $95^{\circ} \mathrm{C}$ in $0.5^{\circ} \mathrm{C}$ increments for $5 \mathrm{~s}$ each) with $2 \times$ iQ SYBR green mix (Bio-Rad) with primers exoL-JGfwd versus exoL-JGrev (Glenn et al. 2007) on $3 \mu \mathrm{l}$ of a $1 / 10$ dilution of each RT reaction. qPCR of $16 \mathrm{~S}$ rRNA was performed under the same conditions with primers JG16Sfwd versus JG16Srev (Gurich and Gonzalez 2009) on a 1/50 dilution of each RT reaction. Negative controls were $3 \mu \mathrm{l}$ of a $1 / 10$ dilution of each mock no-RT control reaction for exoL and $3 \mu \mathrm{l}$ of a 1/50 dilution of each mock no-RT control reaction for $16 \mathrm{~S}$. Expression was quantified by $\mathrm{qPCR}$ with each primer set against a standard dilution curve of an RT reaction performed on $S$. meliloti 1021 total RNA. exoL expression was normalized to $16 \mathrm{~S}$ rRNA expression and the resulting value multiplied by $10^{7}$ for presentation.

Detection of GUS activity and imaging of roots and nodules.

GUS expression by bacteria on root surfaces and within nodules was detected by staining whole roots in X-gluc buffer (1 mM 5-bromo-4-chloro-3-indolyl-beta-D-glucuronic acid, cyclohexylammonium salt; $0.02 \% \mathrm{SDS}$; and $50 \mathrm{mM} \mathrm{Na}$ phosphate, pH 7) (Jefferson et al. 1987) for 23 to $47 \mathrm{~h}$. Whole roots were imaged on an AZ100 Multi-Zoom Microscope equipped with a DS-Fi1, 5 Megapixel color camera (Nikon Instruments U.S., Melville, NY, U.S.A.).

\section{ACKNOWLEDGMENTS}

We thank G. C. Walker (Massachusetts Institute of Technology) for his kind gift of the $\alpha$-ExoK antisera and the reviewers for their comments and suggestions. This work was funded by the United States Department of Agriculture National Institute of Food and Agriculture, Agriculture and Food Research Initiative grant 2010-65108-20582 to K. M. Jones. H. C. Mendis, C. Queiroux, and B. K. Washburn constructed strains, performed experiments, analyzed data, and critically reviewed the manuscript; T. E. Brewer and O. M. Davis constructed strains; and K. M. Jones designed experiments, constructed strains, performed experiments, analyzed data, and wrote the manuscript.

\section{LITERATURE CITED}

Altschul, S. F., Madden, T. L., Schaffer, A. A., Zhang, J., Zhang, Z., Miller, W., and Lipman, D. J. 1997. Gapped BLAST and PSI-BLAST: A new generation of protein database search programs. Nucleic Acids Res. 25:3389-3402.

Aman, P., McNeil, M., Franzen, L.-E., Darvill, A. G., and Albersheim, P. 1981. Structural elucidation, using HPLC-MS and GLC-MS, of the acidic exopolysaccharide secreted by Rhizobium meliloti strain Rm1021. Carbohydr. Res. 95:263-282.

Aslam, S. N., Newman, M. A., Erbs, G., Morrissey, K. L., Chinchilla, D., Boller, T., Jensen, T. T., De Castro, C., Ierano, T., Molinaro, A., Jackson, R. W., Knight, M. R., and Cooper, R. M. 2008. Bacterial polysaccharides suppress induced innate immunity by calcium chelation. Curr. Biol. 18:1078-1083.

Backman, K., and Boyer, H. W. 1983. Tetracycline resistance determined by pBR322 is mediated by one polypeptide. Gene 26:197-203.

Barnett, M. J., Oke, V., and Long, S. R. 2000. New genetic tools for use in the Rhizobiaceae and other bacteria. Biotechniques 29:240-242, 244245 .

Battisti, L., Lara, J. C., and Leigh, J. A. 1992. Specific oligosaccharide form of the Rhizobium meliloti exopolysaccharide promotes nodule invasion in alfalfa. Proc. Natl. Acad. Sci. U.S.A. 89:5625-5629.

Beattie, G. A. 2011. Water relations in the interaction of foliar bacterial pathogens with plants. Annu. Rev. Phytopathol. 49:533-555.
Becker, A., Kleickmann, A., Arnold, W., and Puhler, A. 1993. Analysis of the Rhizobium meliloti exoH/exoK/exoL fragment: ExoK shows homology to excreted endo-beta-1,3-1,4-glucanases and ExoH resembles membrane proteins. Mol. Gen. Genet. 238:145-154.

Bieleski, R. L. 1973. Phosphate pools, phosphate transport, and phosphate availability. Annu. Rev. Plant Physiol. Plant Mol. Biol. 24:225-252.

Borthakur, D., Barber, C. E., Lamb, J. W., Daniels, M. J., Downie, J. A., and Johnston, A. W. B. 1986. A mutation that blocks exopolysaccharide synthesis prevents nodulation of peas by Rhizobium leguminosarum but not of beans by $R$. phaseoli and is corrected by cloned DNA from $R h i$ zobium or the phytopathogen Xanthomonas. Mol. Gen. Genet. 203:320323.

Bradford, M. 1976. A rapid and sensitive method for the quantitation of microgram quantities of protein utilizing the principle of protein-dye binding. Anal. Biochem. 72:248-254.

Buchanan, B. B., Gruissem, W., and Jones, R. L. 2000. Biochemistry and Molecular Biology of Plants. John Wiley and Sons. New York.

Chen, E. J., Sabio, E. A., and Long, S. R. 2008. The periplasmic regulator ExoR inhibits ExoS/ChvI two-component signalling in Sinorhizobium meliloti. Mol. Microbiol. 69:1290-1303.

Cheng, H. P., and Walker, G. C. 1998. Succinoglycan is required for initiation and elongation of infection threads during nodulation of alfalfa by Rhizobium meliloti. J. Bacteriol. 180:5183-5191.

Diebold, R., and Noel, K. D. 1989. Rhizobium leguminosarum exopolysaccharide mutants: Biochemical and genetic analyses and symbiotic behavior on three hosts. J. Bacteriol. 171:4821-4830.

Djordjevic, S. P., Rolfe, B. G., Batley, M., and Redmond, J. W. 1986. The structure of the exopolysaccharide from Rhizobium sp. strain Anu280 (NGR234). Carbohydr. Res. 148:87-99.

Egelhoff, T. T., and Long, S. R. 1985. Rhizobium meliloti nodulation genes: Identification of nodDABC gene products, purification of nodA protein, and expression of nodA in Rhizobium meliloti. J. Bacteriol. 164:591-599.

Fellay, R., Frey, J., and Krisch, H. 1987. Interposon mutagenesis of soil and water bacteria: A family of DNA fragments designed for in vitro insertional mutagenesis of gram-negative bacteria. Gene 52:147-154.

Ferguson, G. P., Datta, A., Carlson, R. W., and Walker, G. C. 2005. Importance of unusually modified lipid A in Sinorhizobium stress resistance and legume symbiosis. Mol. Microbiol. 56:68-80.

Finan, T. M., Hartweig, E., LeMieux, K., Bergman, K., Walker, G. C., and Signer, E. R. 1984. General transduction in Rhizobium meliloti. J. Bacteriol. 159:120-124.

Finan, T. M., Kunkel, B., de Vos, G. F., and Signer, E. R. 1986. Second symbiotic megaplasmid in Rhizobium meliloti carrying exopolysaccharide and thiamine synthesis genes. J. Bacteriol. 167:66-72.

Finan, T. M., Weidner, S., Wong, K., Buhrmester, J., Chain, P., Vorhölter, F. J., Hernandez-Lucas, I., Becker, A., Cowie, A., Gouzy, J., Golding, B., and Pühler, A. 2001. The complete sequence of the 1,683-kb pSymB megaplasmid from the N2-fixing endosymbiont Sinorhizobium meliloti. Proc. Natl. Acad. Sci. U.S.A. 98:9889-9894.

Gage, D. J. 2004. Infection and invasion of roots by symbiotic, nitrogenfixing rhizobia during nodulation of temperate legumes. Microbiol. Mol. Biol. Rev. 68:280-300.

Gage, D. J., Bobo, T., and Long, S. R. 1996. Use of green fluorescent protein to visualize the early events of symbiosis between Rhizobium meliloti and alfalfa (Medicago sativa). J. Bacteriol. 178:7159-7166.

Galibert, F., Finan, T. M., Long, S. R., Pühler, A., Abola, P., Ampe, F., Barloy-Hubler, F., Barnett, M. J., Becker, A., Boistard, P., Bothe, G., Boutry, M., Bowser, L., Buhrmester, J., Cadieu, E., Capela, D., Chain, P., Cowie, A., Davis, R. W., Dreano, S., Federspiel, N. A., Fisher, R. F., Gloux, S., Godrie, T., Goffeau, A., Golding, B., Gouzy, J., Gurjal, M., Hernandez-Lucas, I., Hong, A., Huizar, L., Hyman, R. W., Jones, T., Kahn, D., Kahn, M. L., Kalman, S., Keating, D. H., Kiss, E., Komp, C., Lelaure, V., Masuy, D., Palm, C., Peck, M. C., Pohl, T. M., Portetelle, D., Purnelle, B., Ramsperger, U., Surzycki, R., Thebault, P., Vandenbol, M., Vorhölter, F. J., Weidner, S., Wells, D. H., Wong, K., Yeh, K. C., and Batut, J. 2001. The composite genome of the legume symbiont Sinorhizobium meliloti. Science 293:668-672.

Gibson, K. E., Kobayashi, H., and Walker, G. C. 2008. Molecular determinants of a symbiotic chronic infection. Annu. Rev. Genet. 42:413-441.

Gil-Serrano, A., Sanchez del Junco, A., and Tejero-Mateo, P. 1990. Structure of the extracellular polysaccharide secreted by Rhizobium leguminosarum var. phaseoli CIAT 899. Carbohydr. Res. 204:103-107.

Glazebrook, J., and Walker, G. C. 1989. A novel exopolysaccharide can function in place of the calcofluor-binding exopolysaccharide in nodulation of alfalfa by Rhizobium meliloti. Cell 56:661-672.

Glazebrook, J., and Walker, G. C. 1991. Genetic techniques in Rhizobium meliloti. Methods Enzymol. 204:398-418.

Glenn, S. A., Gurich, N., Feeney, M. A., and Gonzalez, J. E. 2007. The ExpR/Sin quorum-sensing system controls succinoglycan production in 
Sinorhizobium meliloti. J. Bacteriol. 189:7077-7088.

Glucksmann, M. A., Reuber, T. L., and Walker, G. C. 1993a. Family of glycosyl transferases needed for the synthesis of succinoglycan by $R h i$ zobium meliloti. J. Bacteriol. 175:7033-7044.

Glucksmann, M. A., Reuber, T. L., and Walker, G. C. 1993b. Genes needed for the modification, polymerization, export, and processing of succinoglycan by Rhizobium meliloti: A model for succinoglycan biosynthesis. J. Bacteriol. 175:7045-7055.

Gurich, N., and Gonzalez, J. E. 2009. Role of quorum sensing in Sinorhizobium meliloti-alfalfa symbiosis. J. Bacteriol. 191:4372-4382.

Israel, D. W. 1987. Investigation of the role of phosphorus in symbiotic dinitrogen fixation. Plant Physiol. 84:835-840.

Jansson, P.-E., Kenne, L., Lindberg, B., Ljunggren, H., Ruden, U., and Svensson, S. 1977. Demonstration of an octasaccharide repeating unit in the extracellular polysaccharide of $R$. meliloti by sequential degradation. J. Am. Chem. Soc. 99:3812-3815.

Jefferson, R. A., Kavanagh, T. A., and Bevan, M. W. 1987. GUS fusions: Beta-glucuronidase as a sensitive and versatile gene fusion marker in higher plants. EMBO (Eur. Mol. Biol. Organ. J. 6:3901-3907.

Johnson, L. E. B., and Vance, C. P. 1981. Histological and biochemical comparisons of plant induced ineffective nodules. Phytopathology 71:884-884

Jones, K. M. 2012. Increased production of the exopolysaccharide succinoglycan enhances Sinorhizobium meliloti 1021 symbiosis with the host plant Medicago truncatula. J. Bacteriol. 194:4322-4331.

Jones, K. M., Kobayashi, H., Davies, B. W., Taga, M. E., and Walker, G C. 2007. How rhizobial symbionts invade plants: The SinorhizobiumMedicago model. Nat. Rev. Microbiol. 5:619-633.

Jones, K. M., Sharopova, N., Lohar, D. P., Zhang, J. Q., VandenBosch, K. A., and Walker, G. C. 2008. Differential response of the plant Medicago truncatula to its symbiont Sinorhizobium meliloti or an exopolysaccharide-deficient mutant. Proc. Natl. Acad. Sci. U.S.A. 105:704-709.

Kelly, S. J., Muszynski, A., Kawaharada, Y., Hubber, A. M., Sullivan, J. T., Sandal, N., Carlson, R. W., Stougaard, J., and Ronson, C. W. 2013. Conditional requirement for exopolysaccharide in the MesorhizobiumLotus symbiosis. Mol. Plant-Microbe Interact. 26:319-329.

Klein, S., Hirsch, A. M., Smith, C. A., and Signer, E. R. 1988. Interaction of nod and exo Rhizobium meliloti in alfalfa nodulation. Mol. PlantMicrobe Interact. 1:94-100.

Laus, M. C., van Brussel, A. A., and Kijne, J. W. 2005. Exopolysaccharide structure is not a determinant of host-plant specificity in nodulation of Vicia sativa roots. Mol. Plant-Microbe Interact. 18:1123-1129.

Leigh, J. A., and Lee, C. C. 1988. Characterization of polysaccharides of Rhizobium meliloti exo mutants that form ineffective nodules. J. Bacteriol. 170:3327-3332.

Leigh, J. A., Signer, E. R., and Walker, G. C. 1985. Exopolysaccharidedeficient mutants of Rhizobium meliloti that form ineffective nodules. Proc. Natl. Acad. Sci. U.S.A. 82:6231-6235.

Leigh, J. A., Reed, J. W., Hanks, J. F., Hirsch, A. M., and Walker, G. C. 1987. Rhizobium meliloti mutants that fail to succinylate their Calcofluor-binding exopolysaccharide are defective in nodule invasion. Cell 51:579-587.

Long, S., Reed, J. W., Himawan, J., and Walker, G. C. 1988. Genetic analysis of a cluster of genes required for synthesis of the Calcofluor-binding exopolysaccharide of Rhizobium meliloti. J. Bacteriol. 170:42394248

Meade, H. M., Long, S. R., Ruvkun, G. B., Brown, S. E., and Ausubel, F M. 1982. Physical and genetic characterization of symbiotic and auxotrophic mutants of Rhizobium meliloti induced by transposon Tn5 mutagenesis. J. Bacteriol. 149:114-122.

Mendrygal, K. E., and González, J. E. 2000. Environmental regulation of exopolysaccharide production in Sinorhizobium meliloti. J. Bacteriol. 182:599-606.

Newman, M. A., Conradsstrauch, J., Scofield, G., Daniels, M. J., and Dow, J. M. 1994. Defense-related gene induction in Brassica campestris in response to defined mutants of Xanthomonas campestris with altered pathogenicity. Mol. Plant-Microbe Interact. 7:553-563.

Niehaus, K., Kapp, D., and Puhler, A. 1993. Plant defence and delayed infection of alfalfa pseudonodules induced by an exopolysaccharide (EPS I)-deficient Rhizobium meliloti mutant. Planta 190:415-425.

Oldroyd, G. E., and Downie, J. A. 2006. Nuclear calcium changes at the core of symbiosis signalling. Curr. Opin. Plant Biol. 9:351-357.

Oldroyd, G. E., Murray, J. D., Poole, P.S., and Downie, J. A. 2011. The rules of engagement in the legume-rhizobial symbiosis. Annu. Rev. Genet. 45:119-144.

O’Neill, M. A., Darvill, A. G., and Albersheim, P. 1991. The degree of esterification and points of substitution by O-acetyl and O-(3-hydroxybutanoyl) groups in the acidic extracellular polysaccharides secreted by Rhizobium leguminosarum biovars viciae, trifolii, and phaseoli are not related to host range. J. Biol. Chem. 266:9549-9555.
Peck, M. C., Fisher, R. F., and Long, S. R. 2006. Diverse flavonoids stimulate NodD1 binding to nod gene promoters in Sinorhizobium meliloti. J. Bacteriol. 188:5417-5427.

Penmetsa, R. V., and Cook, D. R. 2000. Production and characterization of diverse developmental mutants of Medicago truncatula. Plant Physiol. 123:1387-1398.

Peters, N. K., Frost, J. W., and Long, S. R. 1986. A plant flavone, luteolin, induces expression of Rhizobium meliloti nodulation genes. Science 233:977-980.

Pladys, D., and Vance, C. P. 1993. Proteolysis during development and senescence of effective and plant gene-controlled ineffective alfalfa nodules. Plant Physiol. 103:379-384.

Quandt, J., and Hynes, M. F. 1993. Versatile suicide vectors which allow direct selection for gene replacement in gram-negative bacteria. Gene 127:15-21.

Queiroux, C., Washburn, B. K., Davis, O. M., Stewart, J., Brewer, T. E., Lyons, M. R., and Jones, K. M. 2012. A comparative genomics screen identifies a Sinorhizobium meliloti 1021 sodM-like gene strongly expressed within host plant nodules. BMC Microbiol. 12:74.

Reed, J. W., Capage, M., and Walker, G. C. 1991. Rhizobium meliloti exoG and exoJ mutations affect the ExoX-ExoY system for modulation of exopolysaccharide production. J. Bacteriol. 173:3776-3788.

Reinhold, B. B., Chan, S. Y., Reuber, T. L., Marra, A., Walker, G. C., and Reinhold, V. N. 1994. Detailed structural characterization of succinoglycan, the major exopolysaccharide of Rhizobium meliloti Rm1021. J. Bacteriol. 176:1997-2002.

Reuber, T. L., and Walker, G. C. 1993a. The acetyl substituent of succinoglycan is not necessary for alfalfa nodule invasion by Rhizobium meliloti $\mathrm{Rm} 1021$. J. Bacteriol. 175:3653-3655.

Reuber, T. L., and Walker, G. C. 1993b. Biosynthesis of succinoglycan, a symbiotically important exopolysaccharide of Rhizobium meliloti. Cell 74:269-280.

Robertsen, B. K., Aman, P., Darvill, A. G., McNeil, M., and Albersheim, P. 1981. Host-symbiont interactions: V. The structure of acidic extracellular polysaccharides secreted by Rhizobium leguminosarum and Rhizobium trifolii. Plant Physiol. 67:389-400.

Sambrook, J., Fritsch, E. F., and Maniatis, T. 1982. Molecular Cloning: A Laboratory Manual, 2nd ed. Cold Spring Harbor Laboratory Press, Cold Spring Harbor, NY, U.S.A.

Schlüter, J.-P., Reinkensmeier, J., Barnett, M. J., Lang, C., Krol, E., Giegerich, R., Long, S. R., and Becker, A. 2013. Global mapping of transcription start sites and promoter motifs in the symbiotic alphaproteobacterium Sinorhizobium meliloti 1021. BMC Genomics $14: 156$

Schmeisser, C., Liesegang, H., Krysciak, D., Bakkou, N., Le Quere, A., Wollherr, A., Heinemeyer, I., Morgenstern, B., Pommerening-Roser, A., Flores, M., Palacios, R., Brenner, S., Gottschalk, G., Schmitz, R. A. Broughton, W. J., Perret, X., Strittmatter, A. W., and Streit, W. R. 2009. Rhizobium sp. strain NGR234 possesses a remarkable number of secretion systems. Appl. Environ. Microbiol. 75:4035-4045.

Staehelin, C., Forsberg, L. S., D’Haeze, W., Gao, M. Y., Carlson, R. W. Xie, Z. P., Pellock, B. J., Jones, K. M., Walker, G. C., Streit, W. R., and Broughton, W. J. 2006. Exo-oligosaccharides of Rhizobium sp. strain NGR234 are required for symbiosis with various legumes. J. Bacteriol 188:6168-6178.

Staskawicz, B., Dahlbeck, D., Keen, N., and Napoli, C. 1987. Molecular characterization of cloned avirulence genes from race 0 and race 1 of Pseudomonas syringae pv. glycinea. J. Bacteriol. 169:5789-5794.

Timmers, A. C., Auriac, M. C., and Truchet, G. 1999. Refined analysis of early symbiotic steps of the Rhizobium-Medicago interaction in relationship with microtubular cytoskeleton rearrangements. Development 126:3617-3628.

Udvardi, M., and Poole, P. S. 2013. Transport and metabolism in legumerhizobia symbioses. Annu. Rev. Plant Biol. 64:781-805.

Urzainqui, A., and Walker, G. C. 1992. Exogenous suppression of the symbiotic deficiencies of Rhizobium meliloti exo mutants. J. Bacteriol. 174:3403-3406.

Vance, C. P., Johnson, L. E. B., and Hardarson, G. 1980. Histological comparisons of plant and Rhizobium induced ineffective nodules in alfalfa Physiol. Plant Pathol. 17:167-173.

van Workum, W., van Brussel, A., Tak, T., Wijffelman, C., and Kijne, J. 1995. Ethylene prevents nodulation of Vicia sativa ssp. nigra by exopolysaccharide-deficient mutants of Rhizobium leguminosarum bv. viciae. Mol. Plant-Microbe Interact. 8:278-285.

Vasse, J., de Billy, F., and Truchet, G. 1993. Abortion of infection during the Rhizobium meliloti-alfalfa symbiotic interaction is accompanied by a hypersensitive reaction. Plant J. 4:555-566.

Vincent, J. M. 1970. A Manual for the Practical Study of the Root-Nodule Bacteria. Blackwell, Oxford

Voroshilova, V. A., Demchenko, K. N., Brewin, N. J., Borisov, A. Y., and 
Tikhonovich, I. A. 2009. Initiation of a legume nodule with an indeterminate meristem involves proliferating host cells that harbour infection threads. New Phytol. 181:913-923.

Wang, L. X., Wang, Y., Pellock, B., and Walker, G. C. 1999. Structural characterization of the symbiotically important low-molecular-weight succinoglycan of Sinorhizobium meliloti. J. Bacteriol. 181:6788-6796.

Wells, D. H., and Long, S. R. 2002. The Sinorhizobium meliloti stringent response affects multiple aspects of symbiosis. Mol. Microbiol. 43:11151127.

Wood, P. J. 1980. The interaction of direct dyes with water soluble substituted celluloses and cereal beta-glucans. Ind. Eng. Chem. Prod. Res. Dev. 19:19-23.

York, G. M., and Walker, G. C. 1997. The Rhizobium meliloti exoK gene and prsD/prsE/exsH genes are components of independent degradative pathways which contribute to production of low-molecular-weight succinoglycan. Mol. Microbiol. 25:117-134.

York, G. M., and Walker, G. C. 1998a. The Rhizobium meliloti ExoK and
ExsH glycanases specifically depolymerize nascent succinoglycan chains. Proc. Natl. Acad. Sci. U.S.A. 95:4912-4917.

York, G. M., and Walker, G. C. 1998b. The succinyl and acetyl modifications of succinoglycan influence susceptibility of succinoglycan to cleavage by the Rhizobium meliloti glycanases ExoK and ExsH. J. Bacteriol. 180:4184-4191.

Yu, J., Penaloza-Vazquez, A., Chakrabarty, A. M., and Bender, C. L. 1999. Involvement of the exopolysaccharide alginate in the virulence and epiphytic fitness of Pseudomonas syringae pv. syringae. Mol. Microbiol. 33:712-720.

Yun, M. H., Torres, P. S., El Oirdi, M., Rigano, L. A., Gonzalez-Lamothe, R., Marano, M. R., Castagnaro, A. P., Dankert, M. A., Bouarab, K., and Vojnov, A. A. 2006. Xanthan induces plant susceptibility by suppressing callose deposition. Plant Physiol. 141:178-187.

Zhan, H. J., Levery, S. B., Lee, C. C., and Leigh, J. A. 1989. A second exopolysaccharide of Rhizobium meliloti strain SU47 that can function in root nodule invasion. Proc. Natl. Acad. Sci. U.S.A. 86:3055-3059. 